\title{
Chronic inflammation imposes aberrant cell fate in regenerating epithelia through mechanotransduction
}

\author{
Craig S. Nowell ${ }^{1,7,8}$, Pascal D. Odermatt ${ }^{2}$, Luca Azzolin ${ }^{3}$, Sylke Hohnel ${ }^{4}$, Erwin F. Wagner ${ }^{5}$, Georg E. Fantner ${ }^{2}$, \\ Matthias P. Lutolf ${ }^{4}$, Yann Barrandon ${ }^{6}$, Stefano Piccolo ${ }^{3}$ and Freddy Radtke ${ }^{1,8}$
}

\begin{abstract}
Chronic inflammation is associated with a variety of pathological conditions in epithelial tissues, including cancer, metaplasia and aberrant wound healing. In relation to this, a significant body of evidence suggests that aberration of epithelial stem and progenitor cell function is a contributing factor in inflammation-related disease, although the underlying cellular and molecular mechanisms remain to be fully elucidated. In this study, we have delineated the effect of chronic inflammation on epithelial stem/progenitor cells using the corneal epithelium as a model tissue. Using a combination of mouse genetics, pharmacological approaches and in vitro assays, we demonstrate that chronic inflammation elicits aberrant mechanotransduction in the regenerating corneal epithelium. As a consequence, a YAP-TAZ/ $\beta$-catenin cascade is triggered, resulting in the induction of epidermal differentiation on the ocular surface. Collectively, the results of this study demonstrate that chronic inflammation and mechanotransduction are linked and act to elicit pathological responses in regenerating epithelia.
\end{abstract}

Chronic inflammation is associated with a variety of pathologies in self-renewing epithelial tissues, including impaired wound healing, metaplasia and cancer $^{1-3}$. Indeed, several studies have demonstrated that chronic inflammation is in fact a key driver of aberrant function in epithelial cells ${ }^{4-7}$, although the underlying mechanisms are only beginning to be elucidated. Of particular interest is how stem/progenitor cells in self-renewing epithelia are affected by exposure to a chronic inflammatory environment, especially as aberrant stem cell function is associated with diseases linked to chronic inflammation ${ }^{7-10}$.

Previous studies have shown that chronic inflammation can exert a direct effect on epithelial stem/progenitor cells by secreting soluble factors that regulate key signalling cascades controlling stem cell function ${ }^{11-13}$. However, in addition to regulation by soluble factors such as cytokines and growth factors, stem cells are also regulated by a variety of other microenvironmental cues. In particular, the mechanical properties of tissues can profoundly influence cellular responses and can exert a dominant influence on their response to the mileu of extrinsic factors present in the tissue stroma/niche $\mathrm{e}^{14,15}$.

Mechanical cues, such as elasticity and topography, are heavily influenced by the deposition and organization of extracellular matrix
(ECM) proteins ${ }^{16-19}$, which are secreted by stromal cell types such as tissue-resident fibroblasts ${ }^{16,19}$. Interestingly, a hallmark of many chronic inflammatory conditions is fibrosis, in which excessive ECM deposition occurs in response to persistent inflammation ${ }^{20-22}$. This therefore raises the possibility that at least some of the effects of chronic inflammation may be a consequence of altered mechanical cues downstream of fibrosis. Thus, understanding the link between chronic inflammation and tissue mechanical properties may identify new therapeutic targets.

The corneal epithelium (CE), which forms a protective barrier on the anterior ocular surface, is a clinically relevant example of a self-renewing epithelium in which chronic inflammation is closely associated with abnormal function.

The CE is a stratified epithelium that resides on a relatively simple, avascular stroma and which is maintained during homeostasis and repair by corneal epithelial stem cells ${ }^{23}$ (CESCs). At present, the precise identity of CESCs is unknown as stem-cell-specific markers are lacking. However, label-retaining experiments and functional assays indicate that they reside predominantly, although not exclusively, at the limbus, a junctional zone between the cornea and the conjunctiva $^{24,25}$. Importantly, stem cells isolated from the limbus can

\footnotetext{
${ }^{1}$ Swiss Institute for Experimental Cancer Research (ISREC), Ecole Polytechnique Federale de Lausanne (EPFL), Lausanne, Switzerland 1015, Switzerland. ${ }^{2}$ Laboratory for Bio- and Nano-Instrumentation (LBNI), Institute of Bioengineering (IBI), EPFL, Lausanne, Switzerland 1015, Switzerland. ${ }^{3}$ University of Padua, Department of Molecular Medicine, via G. Colombo 3, 35131 Padova, Italy. ${ }^{4}$ Laboratory of Stem Cell Bioengineering (LSCB), IBI, EPFL, Lausanne, Switzerland 1015, Switzerland. ${ }^{5}$ Genes, Development, and Disease Group, F-BBVA Cancer Cell Biology Programme, National Cancer Research Centre (CNIO), 28029 Madrid, Spain. ${ }^{6}$ Stem Cell Dynamics Laboratory (LDSC), IBI, EPFL, Lausanne, Switzerland 1015, Switzerland. ${ }^{7}$ Present address: United Kingdom Stem Cell Bank (UKSCB), National Institute for Biological Standards and Control (NIBSC), Blanche Lane, South Mimms EN6 3QG, UK.

${ }^{8}$ Correspondence should be addressed to C.S.N. or F.R. (e-mail: craigscott.nowell@epfl.ch or freddy.radtke@epfl.ch)
}

Received 8 September 2015; accepted 18 November 2015; published online 21 December 2015; DOI: 10.1038/ncb3290 
be used clinically to reconstitute the corneal epithelium following injury or disease, either by direct transplantation or by grafting in vitro-expanded cultures of limbal stem cells ${ }^{26}$.

Chronic inflammation has a profound effect on the function of CESCs, and is associated with poor outcome in limbal transplantation $^{27}$ and with conditions such as corneal squamous cell metaplasia (CSCM), in which the CE adopts a keratinizing, skin-like fate ${ }^{28-30}$.

We have previously shown that loss of Notch1 in the mouse CE promotes the development of CSCM specifically during repair ${ }^{31}$. In this model CSCM is induced following remodelling of the underlying corneal stroma by the Notch1-deficient CE and is thus a consequence of changes in the surrounding microenvironment. Interestingly, in other stratified epithelial tissues, such as the skin, Notch signalling has been shown to negatively regulate inflammation ${ }^{32,33}$, raising the possibility that an aberrant inflammatory response during wound healing may play a role in promoting CSCM in Notch1 mutants. In this study, we have used Notch 1 mutant mice as a means to investigate the significance of inflammation during CSCM induction and have specifically explored the link between inflammation and mechanotransduction in mediating cell fate alterations.

\section{RESULTS}

\section{Chronic inflammation promotes CSCM}

To investigate the role of inflammation in CSCM, we used Notch $1^{l o x} /$ lox $: K 5 C r e^{E R T}$ mice, in which tamoxifen-induced cre activity results in conditional deletion of Notch1 in the CE (ref. 31). Consistent with our previous report, the unwounded Notch1-deficient $\left(N o t c h 1^{\Delta}\right)$ cornea maintained corneal identity on the ocular surface, as indicated by the expression of the corneal-specific cytokeratin 12 (K12), and exhibited a simple, relatively acellular stroma, and was thus indistinguishable from wild-type (WT) controls (Supplementary Fig. 1a). However, after repeated injury (Fig. 1a), Notch $1^{\Delta}$ mice developed corneal opacity and significant morphological changes at the histological level (Fig. 1b). Specifically, the Notch $1^{\Delta}$ corneal stroma was infiltrated with large numbers of polymorphonuclear neutrophils, indicative of an ongoing inflammatory response, and exhibited CSCM, indicated by expression of the epidermal-specific cytokeratin 1 (K1; Fig. 1b). In contrast, WT controls did not exhibit any overt signs of inflammation and retained a $\mathrm{K}_{1} 2^{+} \mathrm{K} 1^{-}$corneal epithelium, although $\mathrm{K} 12^{-} \mathrm{K} 1^{+}$cells were present at the limbus (Fig. 1b). Flow cytometric analysis confirmed that Notch $1^{\Delta}$ corneas contained increased numbers of $\mathrm{CD} 45^{+}$leukocytes both during repair and at least 21 days after injury when wound closure had occurred (Fig. 1c,d), with most infiltrating CD $45^{+}$cells exhibiting a $\mathrm{CD} 11 \mathrm{~b}^{+} \mathrm{Gr}^{+}$phenotype (Supplementary Fig. 1b,c), indicative of neutrophils. Furthermore, a variety of pro-inflammatory cytokines exhibited significant upregulation in the Notch $1^{\Delta}$ CE compared with WT CE following injury (Fig. 1e). Collectively, the above findings indicate that CSCM in Notch $1^{\Delta}$ mice is closely associated with an augmented and chronic inflammatory response.

In light of this, we sought to establish whether chronic inflammation is both necessary and sufficient to induce epidermal differentiation on the ocular surface. We therefore employed two approaches. First, we aimed to abrogate the inflammatory response in $N o t c h 1^{\Delta}$ mice to determine whether aberrant inflammation is essential for CSCM in this model. Second, we aimed to establish whether chronic inflammation is sufficient to induce CSCM in a Notch-independent setting.

Regarding the former, we noted that many of the pro-inflammatory cytokines upregulated in the $\operatorname{Notch}^{\Delta}$ CE are targets of the transcription factor AP-1. Furthermore, the Notch $1^{\Delta}$ CE exhibited increased phosphorylation of the AP-1 family member cjun at Ser 73, indicative of activation (Supplementary Fig. 2a). We therefore reasoned that persistent AP-1 activation in the Notch $1^{\Delta}$ CE contributes to the chronic inflammatory response in Notch $1^{\Delta}$ mice, consistent with previous reports ${ }^{34-36}$. We therefore generated Notch $1^{\text {lox } / l o x}: c j u n^{\text {lox } / l o x}: K 5 C r e^{E R T}$ mice and simultaneously ablated Notch1 and cjun by tamoxifen treatment $\left(\operatorname{Notch}^{\Delta}{ }^{\Delta}\right.$ cjun $\left.{ }^{\Delta}\right)$. Following injury, Notch $1^{\Delta}:$ cjun $^{\Delta}$ mice exhibited a significantly reduced inflammatory response compared with $\operatorname{Notch}^{\Delta}$ mutants despite Notch1 ablation (Supplementary Fig. 2b-e) and strikingly retained a $\mathrm{K} 12^{+} \mathrm{K} 1^{-} \mathrm{CE}$ after repeated injury (Supplementary Fig. 2f-h), supporting the hypothesis that chronic inflammation is essential for the development of CSCM in Notch $1^{\Delta}$ mice.

An important caveat to the experiments using the $\operatorname{Notch}^{\Delta}{ }^{\Delta}$ cjun $^{\Delta}$ mice is that AP-1 regulates a variety of cellular functions in addition to inflammation, including differentiation ${ }^{34,37,38}$, and thus an autonomous effect cannot be ruled out. Therefore, to definitively establish that inflammation is the key driver of CSCM in Notch $1^{\Delta}$ mice, we administered a dexamethasone-based anti-inflammatory gel (Tobradex) to the ocular surface during the repeated injury procedure (Fig. 2a). Strikingly, Notch $1^{\Delta}$ mice treated with Tobradex exhibited a relatively normal corneal stroma and maintained a $\mathrm{K} 12^{+} \mathrm{K} 1^{-} \mathrm{CE}$ (Fig. 2b), despite Notch1 ablation (Supplementary Fig. 2i), thus confirming that chronic inflammation is essential for CSCM in Notch $1^{\Delta}$ mutants.

To establish whether chronic inflammation is sufficient for the induction of CSCM in a Notch-independent setting, we performed the repeated injury procedure on K14TSLPTg mice ${ }^{39}$, which develop chronic inflammation in stratified epithelia due to the expression of the pro-inflammatory cytokine TSLP (ref. 40; Fig. 2c). Strikingly, K14TSLPTg mice exhibited a near identical phenotype to Notch $1^{\Delta}$ mutants (Fig. 2d), confirming that chronic inflammation is indeed sufficient for the induction of CSCM.

\section{CSCM is induced in activated stem/progenitor cells located at the limbus and peripheral cornea}

We next sought to address the cellular and molecular mechanism by which chronic inflammation promotes aberrant cell fate and to this end used Notch $1^{\Delta}$ mice as a model of inflammation-associated CSCM.

Initially, we determined the spatial and temporal kinetics of CSCM development in Notch $1^{\Delta}$ mice. Whole-mount immunofluorescence on CE isolated after each injury-repair cycle demonstrated that CSCM first became apparent after the second injury (Supplementary Fig. 3a). This was characterized by the presence of $\mathrm{K}_{12}{ }^{-} \mathrm{K} 1^{+}$cells within the Notch $1^{\Delta}$ CE, particularly at the peripheral cornea in close proximity to the limbus (Supplementary Fig. 3a). In contrast, WT CE remained $\mathrm{K} 12^{+} \mathrm{K} 1^{-}$, although $\mathrm{K} 12^{-} \mathrm{K} 1^{+}$epidermal cells were present in the limbus to varying degrees (Supplementary Fig. 3a).

We therefore chose to analyse the second injury-repair cycle at 0, 6 and $24 \mathrm{~h}$ after injury, to determine when and where CSCM is induced. In WT controls, $\mathrm{K}_{12}{ }^{+} \mathrm{K}^{-}$cells predominated throughout the $\mathrm{CE}$ 


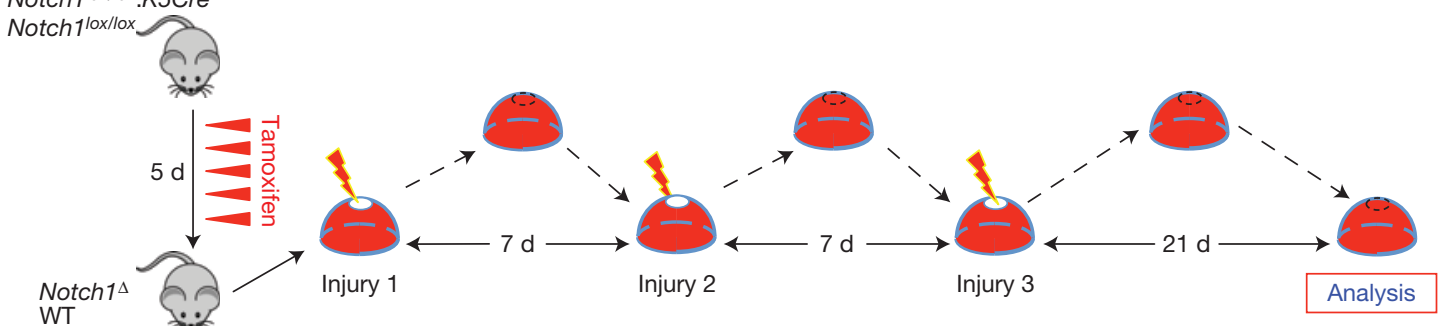

b
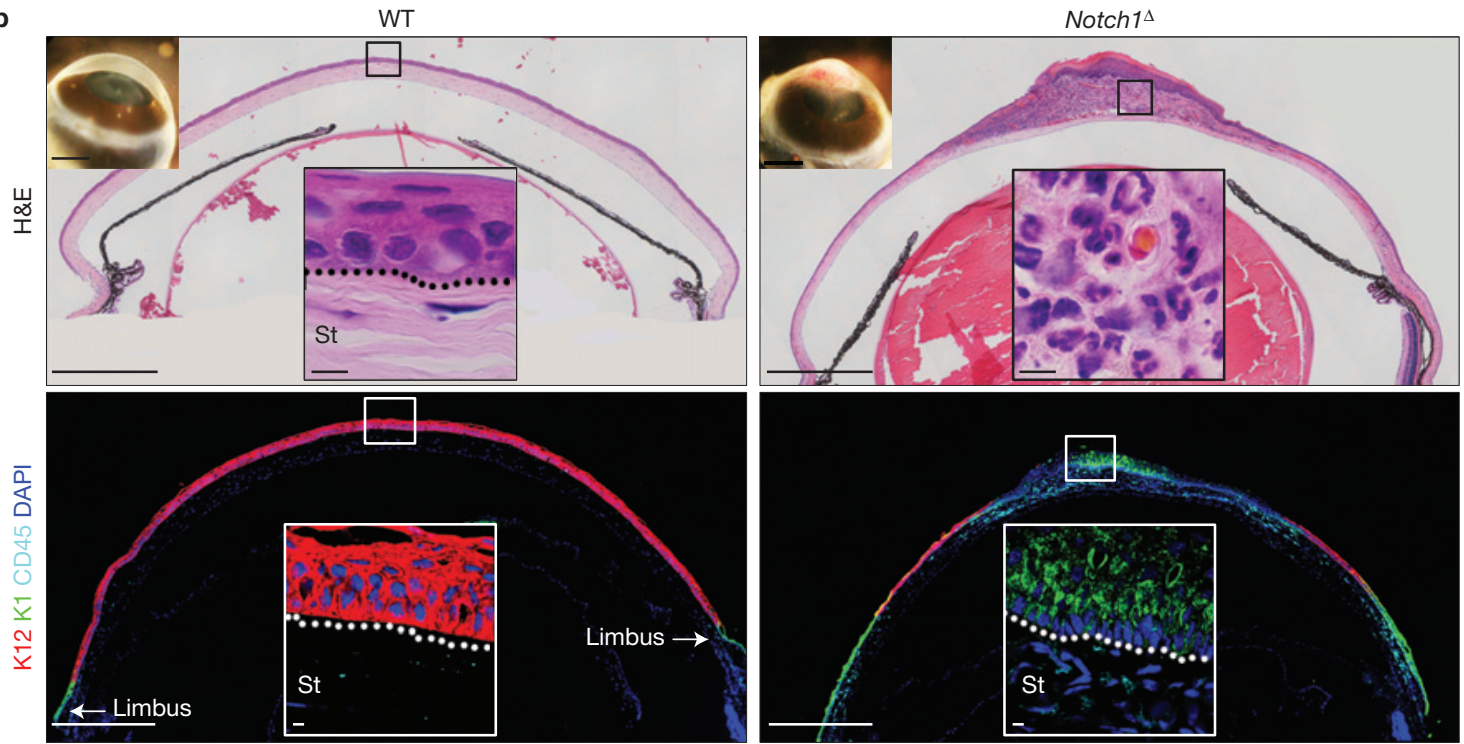
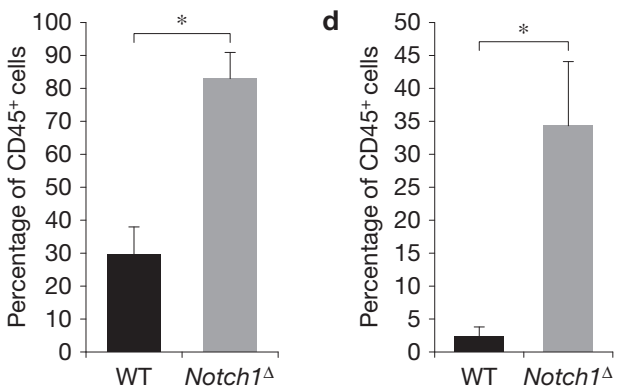

Figure 1 Corneal squamous cell metaplasia (CSCM) in Notch $1^{\Delta}$ mice is associated with an augmented and chronic inflammatory response. (a) Schematic depiction of the experimental strategy. (b) Histology of WT $\left(\right.$ Notch $1^{\text {lox/lox }}$ ) and Notch $1^{\Delta}$ corneal tissue after repeated injury (representative of 16 WT corneas and 20 Notch $1^{\Delta}$ corneas). Upper panels: haematoxylin and eosin (H\&E) staining; lower panels: immunofluorescence for $\mathrm{K} 12, \mathrm{~K} 1$ and $\mathrm{CD} 45$. Large panels are low-magnification tiled images. Insets on the upper left corner of the $\mathrm{H} \& \mathrm{E}$ images show gross phenotype on the ocular surface. Black (H\&E) and white (immunofluorescence) outlined insets show high-magnification images of the indicated regions. (c,d) Quantification of the proportion of CD45 $5^{+}$cells in WT and Notch $1^{\Delta}$ corneas $24 \mathrm{~h}$ after a single injury (c) and 21 days after repeated corneal injury (d). Proportions were measured by performing flow cytometry on dissociated corneas $(n=6$

at each stage of repair, although $\mathrm{K} 12^{-} \mathrm{K} 1^{+}$cells were present in the limbus (Fig. 3a,b), consistent with the observations from whole-mount analysis (Supplementary Fig. 3a). In contrast, Notch $1^{\Delta}$ mice exhibited increasingly prominent areas of $\mathrm{K}_{1} 2^{-} \mathrm{K}^{+}$cells extending from the limbus into the peripheral cornea as wound healing progressed (Fig. 3a,b). In addition, the epithelium in the limbus and peripheral

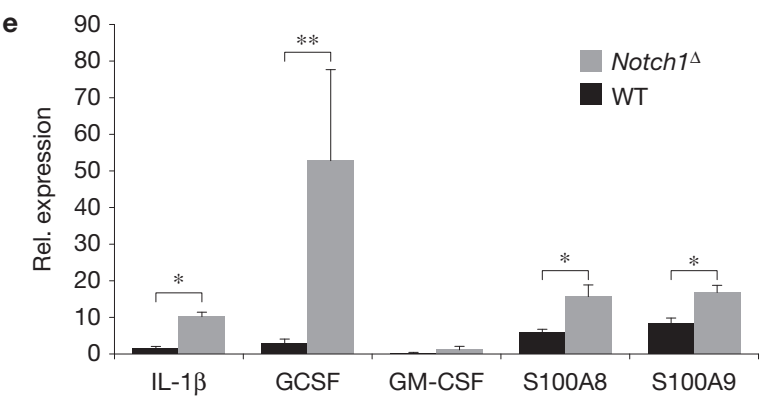

biological replicates for each genotype over three independent experiments; each replicate consists of cells pooled from 4 corneas isolated from 2 mice of each genotype). (e) QRT-PCR analysis for the indicated cytokines in WT (Notch $1^{\text {lox/lox }}$ ) and Notch $1^{\Delta}$ corneal epithelial cells $24 \mathrm{~h}$ after a single corneal injury. Data are expressed relative to the expression in WT unwounded corneal epithelial cells ( $n=6$ biological replicates for each genotype over three independent experiments; each replicate consists of corneal epithelial tissue pooled from 6 corneas isolated from 3 mice of each genotype). Scale bars represent $500 \mu \mathrm{m}$ on tiled images and $5 \mu \mathrm{m}$ on all other histological images. Scale bars on images showing gross morphology of corneas represent $1 \mathrm{~mm}$. Black and white dotted lines indicate the boundary between the stroma and epithelium. St, stroma. ${ }^{*} P<0.01,{ }^{* *} P<0.05$ (unpaired, two-tailed $t$-tests). Error bars represent standard deviation.

cornea of $\operatorname{Notch}^{\Delta}{ }^{\Delta}$ mice was generally less stratified than in WT controls (Fig. 3a). Consistent with the whole-mount analysis, no overt phenotypic differences were apparent between WT and Notch $1^{\Delta} \mathrm{CE}$ during the first injury-repair cycle (Supplementary Fig. 3b).

The above findings indicate that CSCM in $\operatorname{Notch}^{\Delta}$ mice is initially induced at the limbus/peripheral cornea and subsequently 


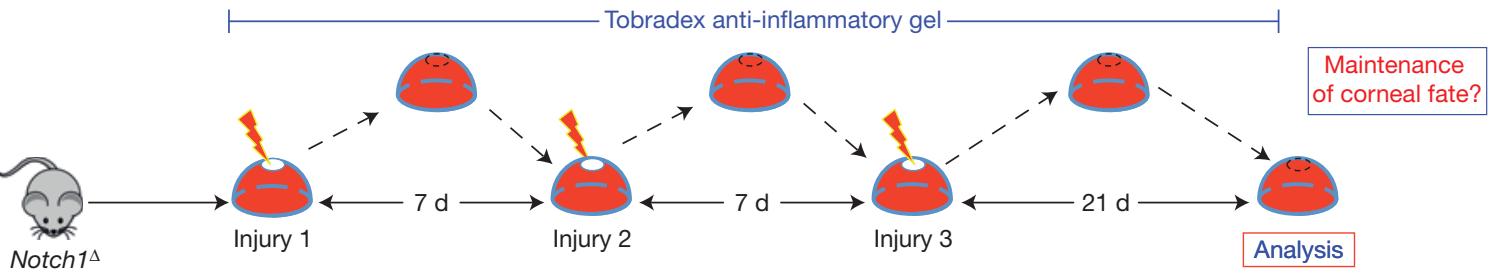

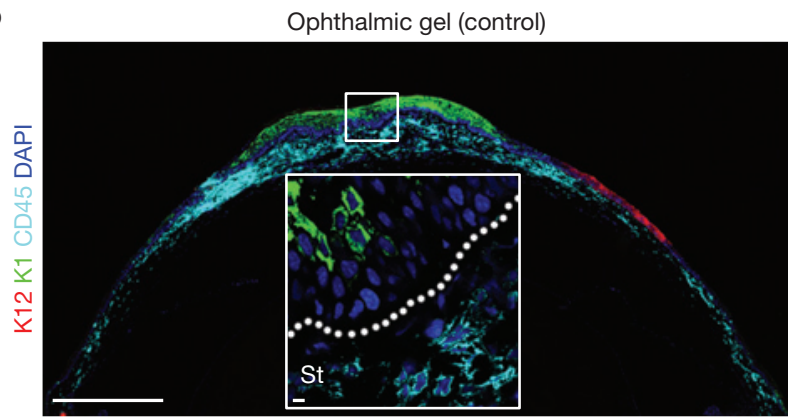
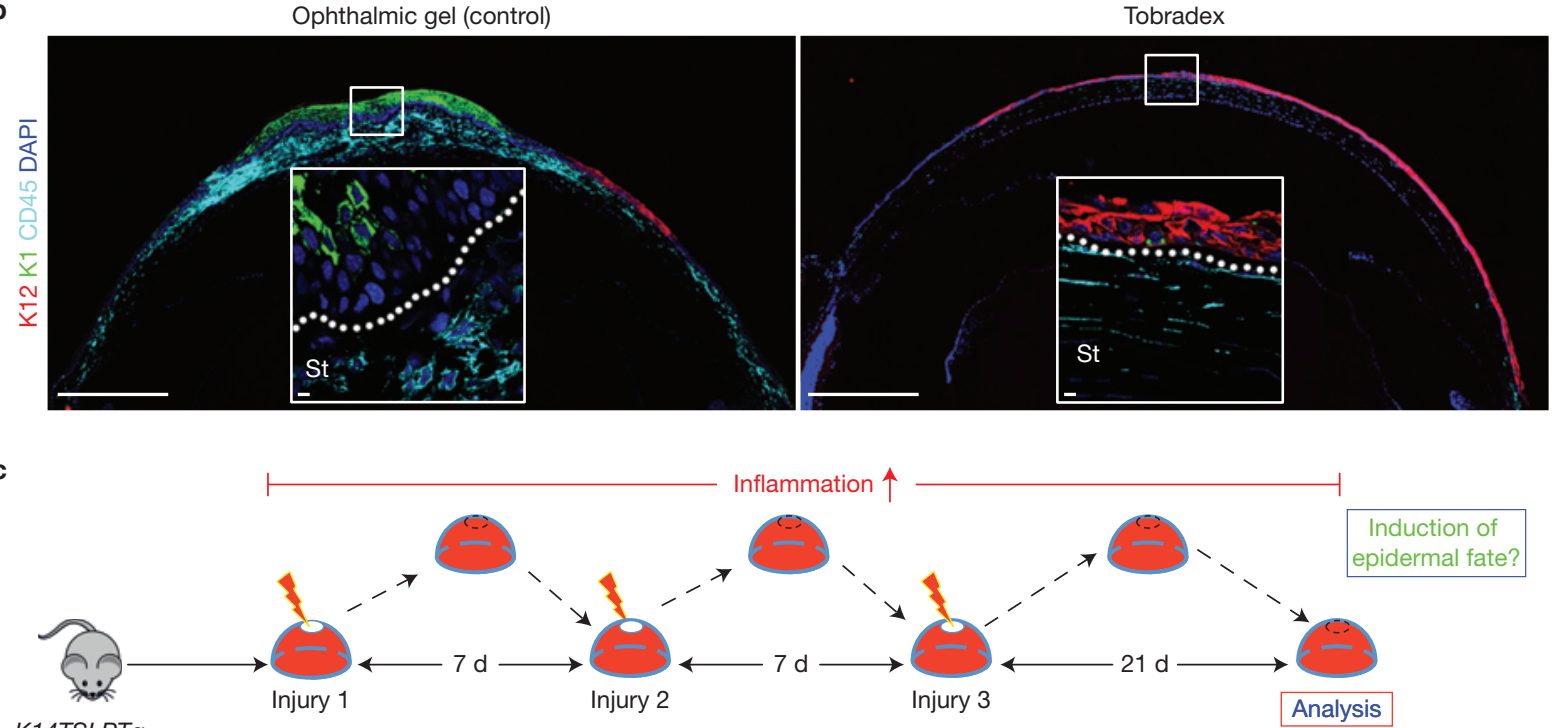

K14TSLPTg

WT

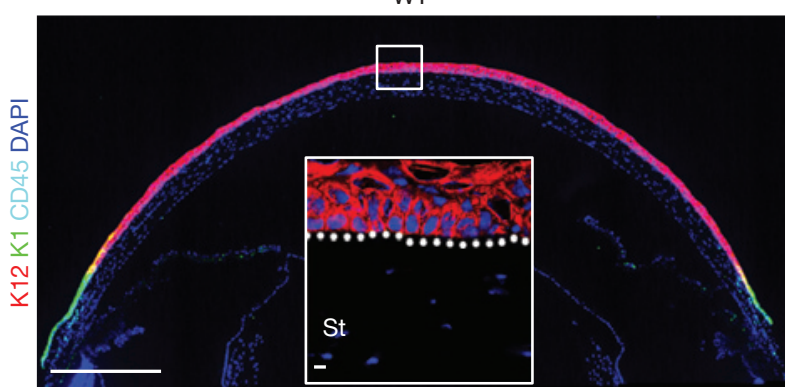

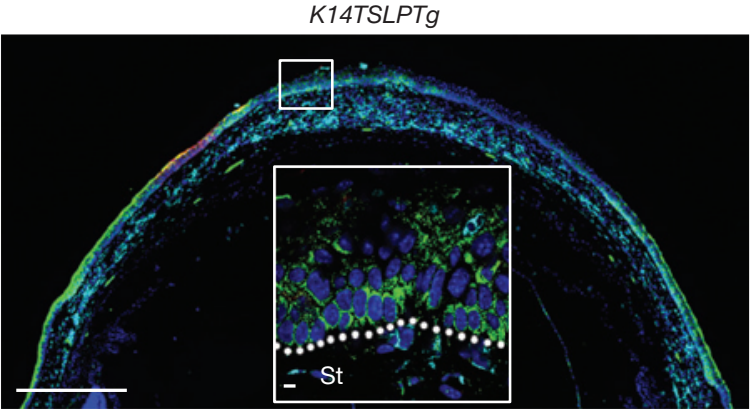

Figure 2 Chronic inflammation is necessary and sufficient to induce CSCM. (a) Schematic depiction of the experimental strategy used to determine whether chronic inflammation is necessary for the induction of CSCM in Notch $1^{\Delta}$ mice. (b) Immunofluorescent staining for K12, K1 and CD45 on Notch $1^{\Delta}$ corneas treated with ophthalmic gel (control) or the antiinflammatory gel Tobradex. The images are representative of 6 corneas per treatment over three independent experiments. Large panels are lowmagnification tiled images. Insets show high-magnification images of the indicated regions. (c) Schematic depiction of the experimental strategy

migrates to the site of injury as wound closure proceeds. This is consistent with the model of corneal regeneration proposed in a previous study, which found that cells mediating wound healing are derived from stem/progenitors located in the limbus ${ }^{25}$. In support of this, we observed increased proliferative activity in the limbus and peripheral cornea following injury (Supplementary Fig. $3 c, d$ ), suggesting activation of stem/progenitor cells specifically within these regions. In addition, whole-mount immunofluorescence on $\mathrm{CE}$ isolated after repeated injury revealed that metaplastic $\mathrm{K} 12^{-} \mathrm{K}^{+}$regions in $\operatorname{Notch}^{\Delta}$ CE remained continuous with the used to determine whether chronic inflammation is sufficient to induce CSCM. (d) Immunofluorescent staining for K12, K1 and CD45 on WT (nontransgenic littermates) and K14TSLPTg corneas after the procedure shown in c. The images are representative of 6 corneas per genotype over three independent experiments. Large panels are low-magnification tiled images. Insets show high-magnification images of the indicated regions. Scale bars represent $500 \mu \mathrm{m}$ on tiled images and $5 \mu \mathrm{m}$ on all other images. White dotted lines indicate the boundary between the stroma and epithelium. St, stroma.

limbus/peripheral cornea, and thus conformed to the pattern expected if CSCM derived from this region (Fig. 3c,d).

\section{Chronic inflammation induces CSCM through elevation of $\beta$-catenin signalling in the CE}

At the molecular level, aberrant Wnt/ $\beta$-catenin signalling has been linked to squamous cell metaplasia in a variety of epithelial tissues, including the cornea ${ }^{41-43}$, and is therefore a good candidate for mediating CSCM in response to chronic inflammation. Consistent with this, the $\operatorname{Notch}^{\Delta} \mathrm{CE}$ exhibited increased expression of $\beta$-catenin 
a
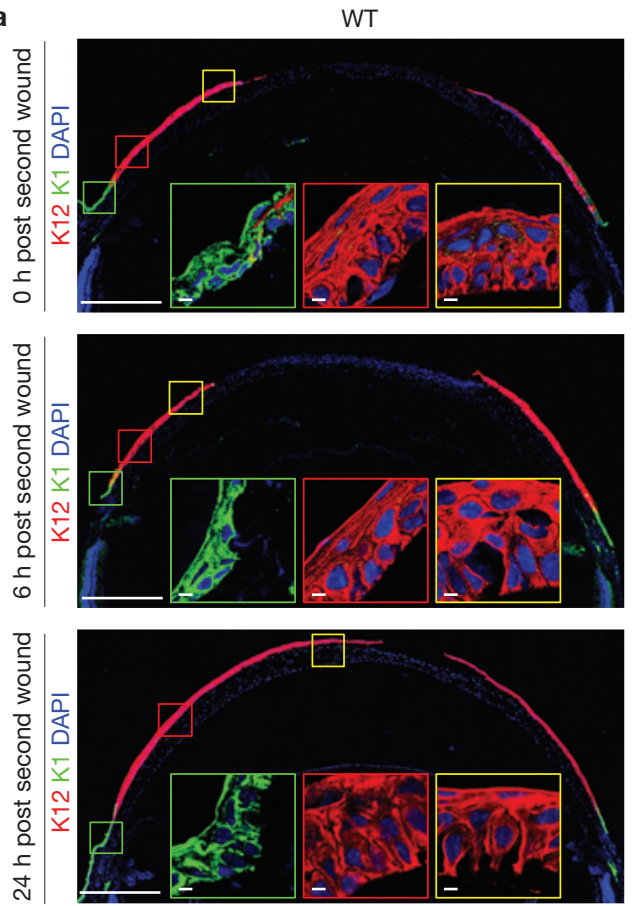

Injury at central cornea

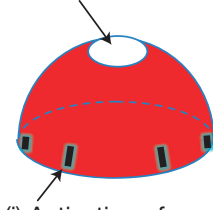

(i) Activation of stem/progenitor cells in limbus/peripheral cornea
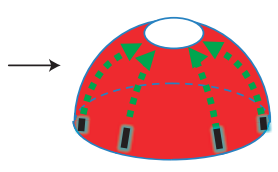

(ii) Epidermal differentiation of progenitor cells as they migrate to close wound
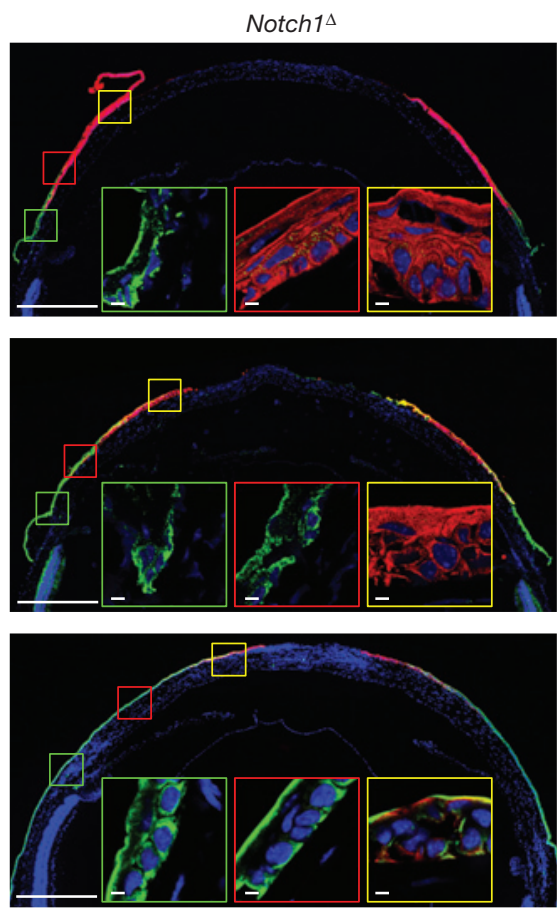

d
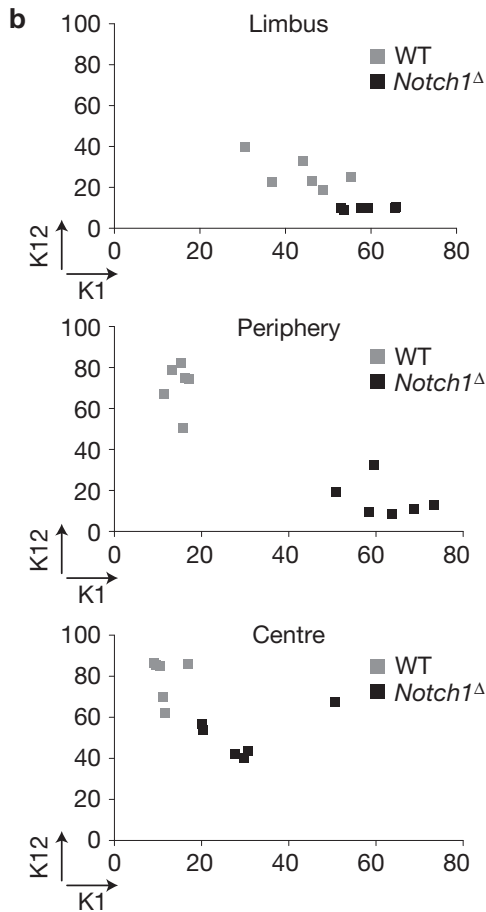

21 days post third wound
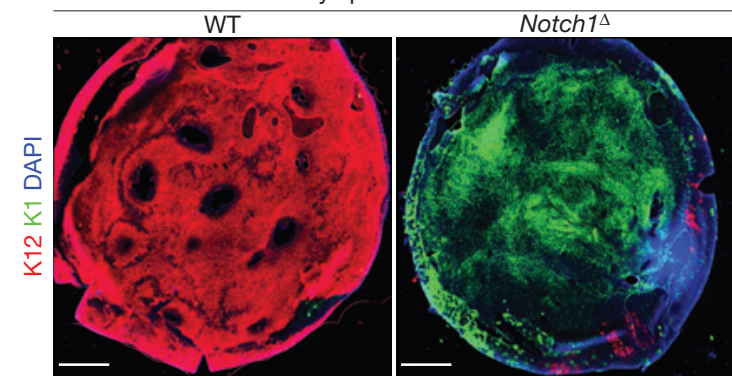

Figure $3 \mathrm{CSCM}$ is induced in limbal and peripheral cells during repair. (a) Immunofluorescent staining for $\mathrm{K} 12$ and $\mathrm{K} 1$ in WT (Notch $1^{\text {lox/lox }}$ ) and Notch ${ }^{\Delta}$ corneas at 0,6 and $24 \mathrm{~h}$ after a second corneal injury. The images are representative of 6 corneas per genotype over two independent experiments for each time point analysed. Large panels are low-magnification tiled images. Insets outlined in green, red and yellow show high-magnification images of the limbus, peripheral cornea and central cornea, respectively. (b) $X Y$ scatter plots showing $\mathrm{K} 12$ and $\mathrm{K} 1$ expression in the limbus, peripheral cornea and central cornea $24 \mathrm{~h}$ after a second corneal injury. Each data point represents mean fluorescence intensity measured from an individual cornea. Grey squares, WT (Notch $1^{\text {lox/lox }}$ ); black squares, Notch $1^{\Delta}(n=6$ corneas for each genotype over three independent experiments). (c) Model predicting the cellular

following induction of CSCM by repeated injury (Fig. 4a). To determine whether increased $\beta$-catenin expression overlapped with CSCM induction, we quantified its expression during each injury-repair cycle. In unwounded corneas and during the first injury-repair cycle, $\beta$-catenin expression was relatively high in the limbus but declined in the peripheral and central CE in WT and Notch $1^{\Delta}$ mice (Supplementary Fig. 4a,b). However, following the second injury, high $\beta$-catenin expression was evident throughout the $N o t c h 1^{\Delta}$ CE (Fig. 4b,c). In WT controls, $\beta$-catenin expression was increased in the limbus, but again declined in the peripheral and central CE (Fig. 4b,c). Thus, high $\beta$-catenin expression origin of CSCM. Following injury to the central cornea, stem/progenitor cells in the peripheral cornea become activated and proliferate to generate daughter cells that mediate wound closure. In Notch $1^{\Delta}$ mutants, chronic inflammation promotes epidermal differentiation of activated stem/progenitor cells or their immediate progeny. This model therefore predicts that all epidermal lineage cells in Notch $1^{\Delta}$ corneas will be derived from peripheral stem/progenitor cells and therefore be continuous with the peripheral cornea. (d) Immunofluorescent staining for K12 and K1 on whole-mount corneal epithelial tissue from WT (Notch $1^{\text {lox/lox }}$ ) and Notch $1^{\Delta}$ mice after repeated injury. The images are representative of 12 corneal whole-mounts per genotype over four independent experiments. Images shown are lowmagnification tiled images. Scale bars represent $500 \mu \mathrm{m}$ on tiled images and $5 \mu \mathrm{m}$ on all other images.

overlapped spatially and temporally with the presence of $\mathrm{K}^{2} 2^{-} \mathrm{K} 1^{+}$ epidermal cells.

To definitively establish whether $\beta$-catenin is the molecular factor that induces CSCM, we performed loss- and gain-offunction experiments in vivo. Thus, to determine whether $\beta$-catenin is necessary for CSCM induction, tamoxifen-treated Notch1 $1^{\text {lox } / \text { lox }}:$ Ctnnb $1^{\text {lox } / \text { lox }}: K 5 C r e^{E R T}$ mice, in which Notch1 and Ctnnb1 $\left(\beta\right.$-catenin) are simultaneously ablated in the CE $\left(N o t c h 1^{\Delta}: \operatorname{Ctnnb1}^{\Delta}\right)$, were subjected to repeated corneal injury (Fig. $4 \mathrm{~d}$ ). This resulted in chronic inflammation in the corneal stroma in a similar manner to $N o t c h 1^{\Delta}$ mutants (Fig. 4e). However, $\beta$-catenin-deficient cells 


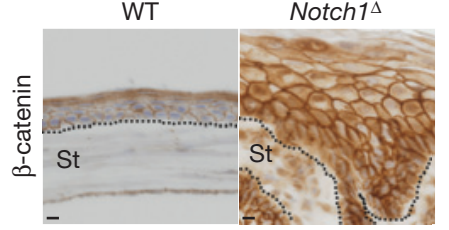

c

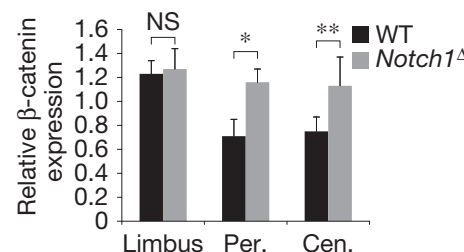

d Notch1lox/lox:Ctnnb1/ox/lox. K5Cre ERT

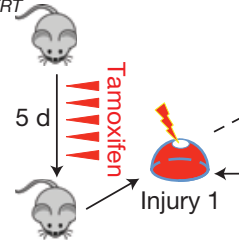

Notch $1^{\Delta}:$ Ctnnb $^{1}$
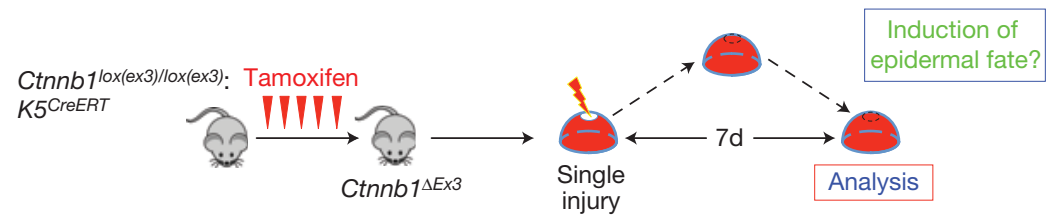

b

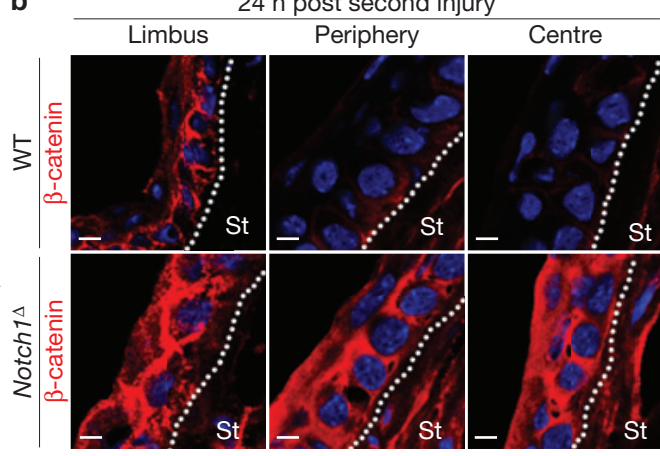

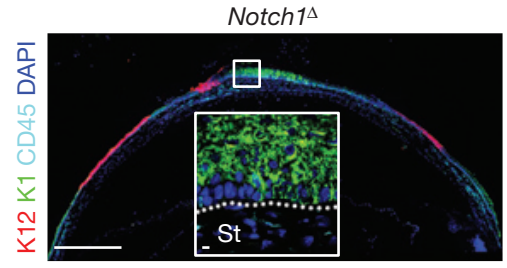

Notch ${ }^{\Delta}:$ :Ctnnb $1 \Delta$

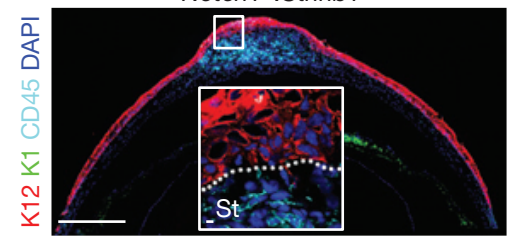

g

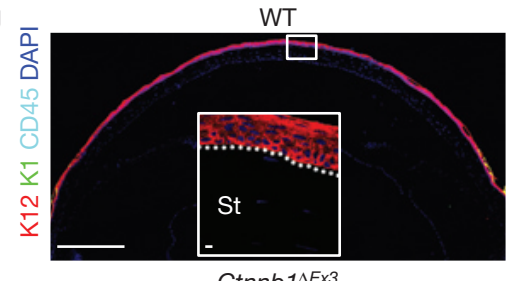

Ctnnb1 ${ }^{\triangle E \times 3}$

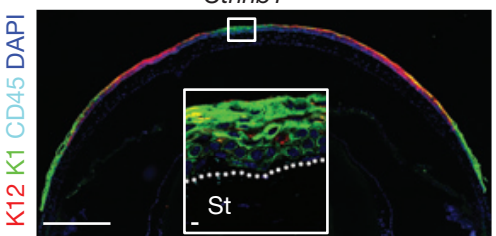

Figure 4 Chronic inflammation promotes CSCM through elevated $\beta$-catenin signalling. (a) Immunohistochemistry for $\beta$-catenin on WT (Notch lox/lox $^{2}$ ) and Notch $1^{\Delta}$ corneas after repeated injury. Data are representative of 8 corneas per genotype over three independent experiments. (b) Immunofluorescent staining for $\beta$-catenin in limbus, peripheral cornea (Per.) and central cornea (Cen.) of WT (Notch ${ }^{\text {lox/lox}}$ ) and Notch $1^{\Delta}$ corneas $24 \mathrm{~h}$ after the second corneal injury. The images are representative of 6 corneas isolated over three independent experiments. (c) Quantification of relative $\beta$-catenin expression in limbus, peripheral cornea and central cornea $24 \mathrm{~h}$ after the second corneal injury. Black bars, WT (Notch $\left.1^{\text {lox/lox }}\right)$; grey bars, Notch $1^{\Delta}(n=6$ corneas for each genotype over three independent experiments). Values for expression levels are relative values normalized to the expression level in the conjunctiva of each sample, determined by mean fluorescence intensity. (d) Schematic depiction of the experimental strategy used to determine whether $\beta$-catenin is necessary for the induction of CSCM. (e) Immunofluorescent staining

retained a predominantly $\mathrm{K} 12^{+} \mathrm{K} 1^{-}$phenotype, even in the presence of a chronic inflammatory environment, although the epithelium remained hyperplastic (Fig. 4e, Supplementary Fig. 4c). Thus, elevated $\beta$-catenin signalling in corneal epithelial cells is essential for inflammation-induced CSCM.

In the reciprocal gain-of-function experiment, we induced constitutive activation of $\beta$-catenin in the $\mathrm{CE}$ using tamoxifentreated $C t n n b 1^{\operatorname{lox}(e \times 3) / \operatorname{lox}(e \times 3)}: K 5 C r e^{E R T}$ mice $^{44}$ (hereafter referred to as $C t n n b 1^{\Delta E x 3}$ mice), in which $\beta$-catenin is stabilized owing to cremediated excision of the degradation domain within exon 3. After a single injury-repair cycle (Fig. 4f), $C \operatorname{Cnn} b 1^{\Delta E x 3}$ mice exhibited epidermal differentiation on the ocular surface, in contrast to WT controls that, as expected, maintained a corneal phenotype (Fig. $4 \mathrm{~g}$ ). In this system, there was no evidence of chronic inflammation, as the presence of $\mathrm{CD}_{4} 5^{+}$cells in the $C \operatorname{tnn} b 1^{\Delta E x 3}$ stroma seemed equivalent to for $\mathrm{K} 12, \mathrm{~K} 1$ and $\mathrm{CD} 45$ on Notch $1^{\Delta}$ and Notch $1^{\Delta}:$ Ctnnb $^{\Delta}$ corneas after the procedure outlined in $\mathbf{d}$. The images are representative of 8 corneas per genotype over three independent experiments. Large panels are lowmagnification tiled images. Insets are high-magnification images of the indicated regions. (f) Schematic depiction of the experimental strategy used to determine whether elevated $\beta$-catenin is sufficient to induce CSCM. (g) Immunofluorescent staining for K12, K1 and CD45 on WT

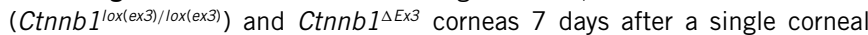
injury. The images are representative of 8 WT corneas and 10 Ctnnb1 ${ }^{\Delta E \times 3}$ corneas over four independent experiments. Large panels are low magnification tiled images. White outlined insets are high-magnification images of the indicated regions. Scale bars represent $500 \mu \mathrm{m}$ on tiled images and $5 \mu \mathrm{m}$ on all other images. White dotted lines indicate the boundary between the stroma and epithelium. St, stroma; NS, not significant. ${ }^{*} P<0.01,{ }^{* *} P<0.05$ (unpaired, two-tailed $t$-tests). Error bars represent standard deviation.

WT controls (Fig. 4g). Furthermore, the induction of epidermal differentiation correlated strictly with elevated $\beta$-catenin expression (Supplementary Fig. 4d). Together, these data strongly suggest that overexpression of $\beta$-catenin is sufficient for the cell-autonomous induction of CSCM. In addition, whole-mount immunofluorescence demonstrated that regions of CSCM in $C t n n b 1^{\triangle E x 3}$ cornea remained continuous with the limbus/peripheral cornea (Supplementary Fig. 4e), thus supporting the hypothesis that CSCM is induced at this location.

\section{Chronic inflammation is associated with excessive ECM deposition, increased tissue stiffness and elevated mechanotransduction in the $\mathrm{CE}$}

We next addressed the mechanism by which chronic inflammation elicits elevated $\beta$-catenin in the CE. Surprisingly, we could not find any evidence of increased Wnt ligand expression in the 
a

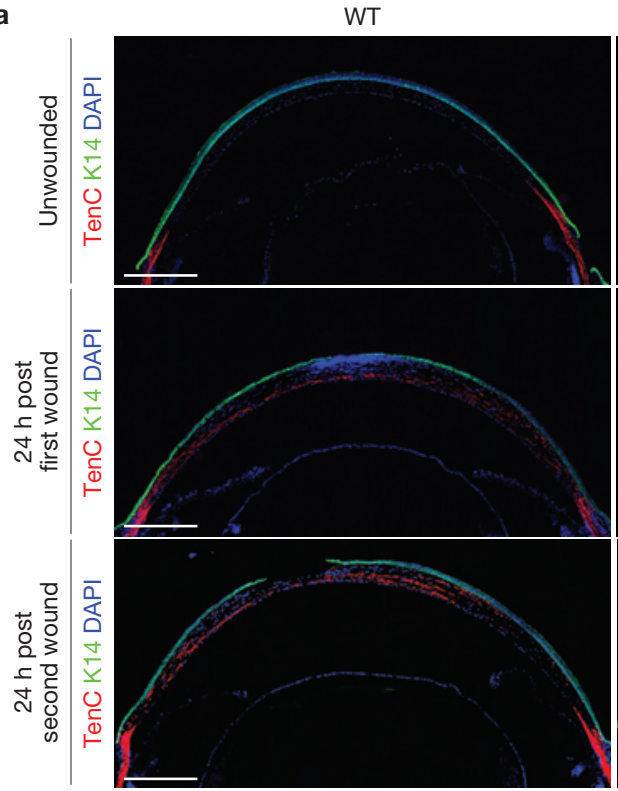

c

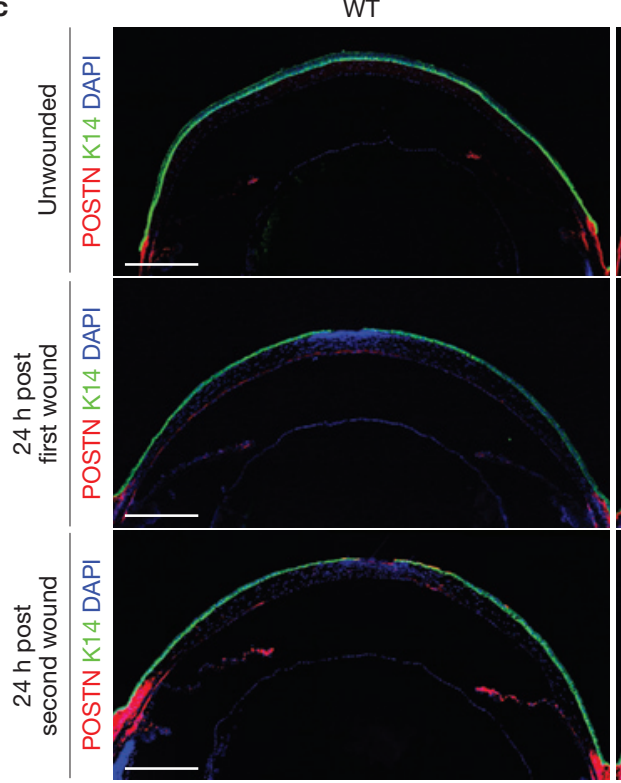

Notch14
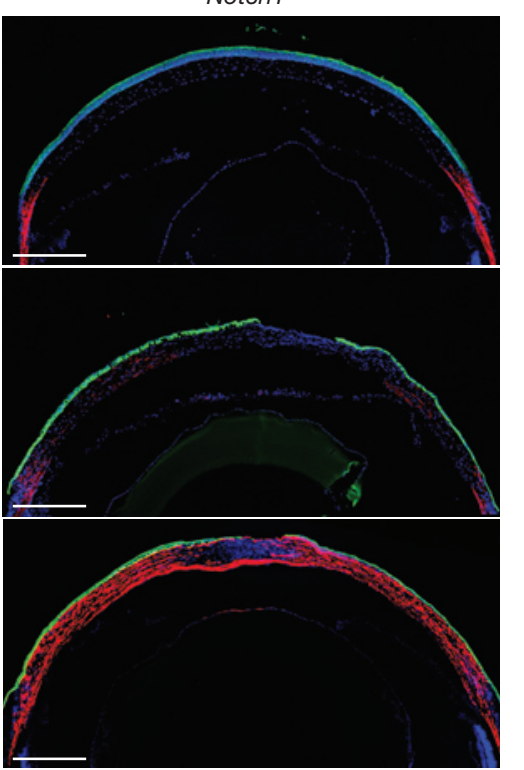

Notch14
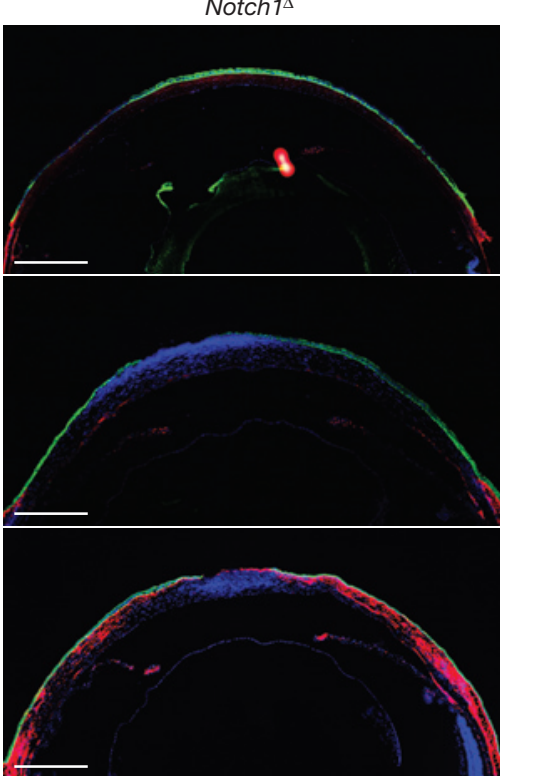

b
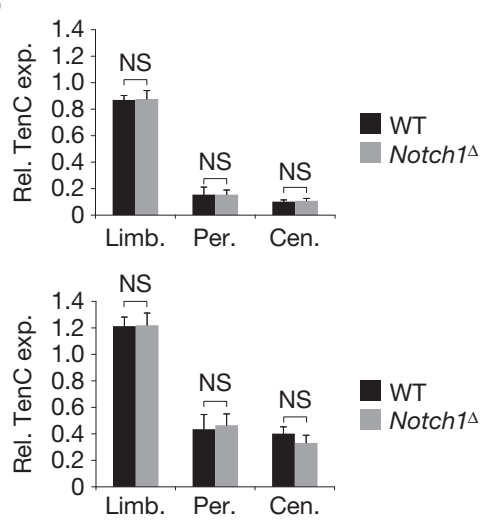

WT

Notch1 ${ }^{4}$

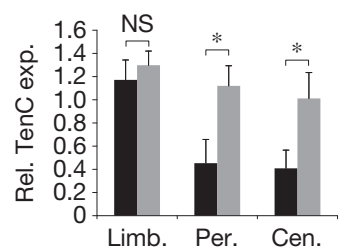

WT

Notch $1^{\perp}$

d
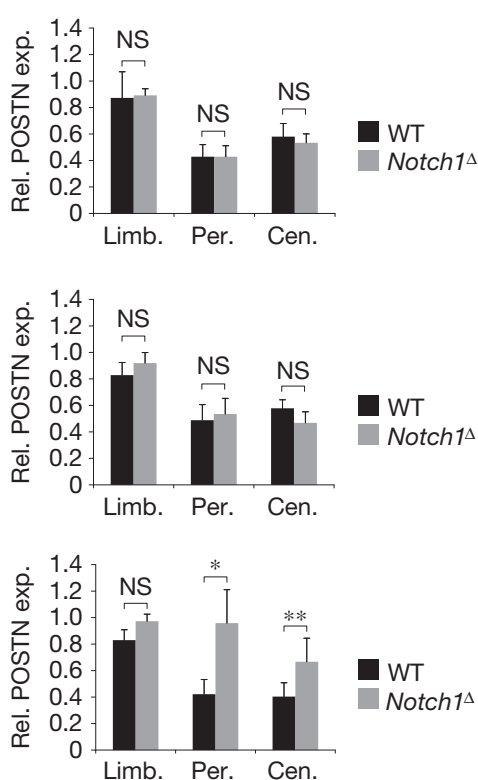

Figure 5 Increased ECM deposition in the corneal stroma in response to aberrant inflammation. (a) Immunofluorescent staining for $\mathrm{K} 14$ and tenascin C (TenC) in WT (Notch $1^{\text {lox/lox }}$ ) and Notch $1^{\Delta}$ corneas, unwounded and $24 \mathrm{~h}$ after first and second wounds. The images are representative of 6 corneas per genotype for each time point analysed over three independent experiments. (b) Relative quantification of tenascin $C$ expression in the limbus (Limb.), peripheral cornea (Per.) and central cornea (Cen.). Upper panel shows uninjured cornea; middle panel shows corneal tissue $24 \mathrm{~h}$ after the first injury; lower panel show corneal tissue $24 \mathrm{~h}$ after the second injury. Values for expression levels are relative values normalized to the expression level in the conjunctival stroma of each sample, determined by mean fluorescence intensity. Black bars, WT (Notch $1^{\text {lox/lox }}$; grey bars, Notch $1^{\Delta}$ ( $n=6$ corneas for each genotype at each time point over three independent

Notch $1^{\Delta}$ cornea (Supplementary Fig. 5a), suggesting that the chronic inflammatory environment does not activate the Wnt signalling cascade. Previous studies have linked mechanotransduction to the induction of $\beta$-catenin activity in epithelia ${ }^{15}$. The mechanisms experiments). (c) Immunofluorescent staining for $\mathrm{K} 14$ and periostin (POSTN) in WT (Notch $1^{\text {lox/lox }}$ ) and Notch $1^{\Delta}$ corneas. The images are representative of 6 corneas per genotype for each time point analysed over three independent experiments. (d) Relative quantification of periostin expression in the limbus, peripheral cornea and central cornea in uninjured tissue (upper panel), corneal tissue $24 \mathrm{~h}$ after the first injury (middle panel) and corneal tissue $24 \mathrm{~h}$ after the second injury (lower panel). Values for expression levels are relative values normalized to the expression level in the conjunctival stroma of each sample, determined by mean fluorescence intensity. $n=6$ corneas for each genotype at each time point over three independent experiments. Scale bars on tiled images represent $500 \mu \mathrm{m}$. NS, not significant. ${ }^{*} P<0.01,{ }^{*} P<0.05$ (unpaired, two-tailed $t$-tests). Error bars represent standard deviation.

underlying mechanotransduction remain to be fully elucidated, although YAP/TAZ have recently emerged as primary cellular sensors of tissue mechanical cues ${ }^{14,45}$. Intriguingly, a recent report has shown that YAP/TAZ directly regulate $\beta$-catenin expression by forming 
an integral part of the cytoplasmic $\beta$-catenin destruction complex. According to the proposed model, removal of YAP/TAZ from the destruction complex, through mechanically induced nuclear translocation, increases $\beta$-catenin expression owing to impaired degradation ${ }^{46}$. We therefore speculated that chronic inflammation may induce aberrant mechanotransduction in the CE by causing changes in the mechanical properties of the corneal stroma, for example through excessive deposition of ECM, as occurs in fibrosis ${ }^{47}$. This would therefore result in nuclear localization of YAP/TAZ and increased $\beta$-catenin expression.

To determine whether increased ECM deposition was associated with CSCM, the expression of the ECM proteins periostin and tenascin $\mathrm{C}$, which are linked to fibrosis ${ }^{48,49}$, was analysed during each injury-repair cycle. In WT corneas, both periostin and tenascin $\mathrm{C}$ were restricted to the stroma underlying the limbus at each stage (Fig. 5a-d). A similar pattern was observed in unwounded Notch ${ }^{\Delta}$ corneas and in $\operatorname{Notch}^{\Delta}$ corneas during the first injuryrepair cycle (Fig. 5a-d). However, expression in the peripheral and central corneal stroma of Notch $1^{\Delta}$ mutants increased markedly during the second injury-repair cycle (Fig. 5a-d). In support of a fibrotic-like response, the expression of periostin and tenascin $\mathrm{C}$ was largely restricted to non-haematopoietic cell types (Supplementary Fig. 5b). Furthermore, abrogation of inflammation in Notch $1^{\Delta}$ mice by Tobradex treatment resulted in reduced ECM deposition in the corneal stroma (Supplementary Fig. 5c), indicating that the effect occurred downstream of inflammation.

ECM deposition in the corneal stroma also correlated with activation of mechanotransduction in the overlying CE after the second injury, as demonstrated by increased phosphorylation of focal adhesion kinase (pFAK), and increased nuclear localization of Rho coiled-coil kinase 2 (ROCK2) and YAP/TAZ (Fig. 6a-g). Importantly, in unwounded corneas and in corneas isolated after the first injury, mechanotransduction was restricted to the ECMrich limbus and was not apparent in the CE (Supplementary Fig. 6a,b). Furthermore, abrogation of inflammation in Notch $1^{\Delta}$ mice by Tobradex treatment reduced mechanotransduction in the corneal epithelium (Supplementary Fig. 6c). Thus, in a similar manner to $\beta$-catenin, there was a spatial and temporal overlap between increased ECM deposition, mechanotransduction and CSCM induction.

To gather additional evidence of a role for mechanotransduction, we determined whether ECM deposition resulted in changes in the mechanical properties of the corneal stroma by employing atomic force microscopy in combination with immunofluorescence. This enabled us to correlate the expression of ECM with tissue stiffness and revealed that increased expression of tenascin C in Notch $1^{\Delta}$ corneas correlated with increased stiffness of the corneal stroma compared with WT controls, an effect that was apparent at the limbus, peripheral $\mathrm{CE}$ and central CE (Fig. 7a,b).

In light of the above data, we sought to establish a functional link between mechanotransduction and $\beta$-catenin signalling. We therefore obtained primary cultures of pig corneal epithelial stem cells (PCESCs), which can be readily cultivated in vitro, and determined the effect of mechanical stimuli by growing these cells on stiff versus soft substrates. Importantly, in this assay, PCESCs were cultured in the absence of feeder cells to promote differentiation.
Strikingly, cells grown on stiff substrates expressed higher levels of $\beta$-catenin compared with cells grown on soft substrates, as well as exhibiting increased nuclear localization of YAP/TAZ and perturbed differentiation (Fig. 7c-g). Moreover, PCESCs cultured on extremely stiff substrates (glass) in the presence of the ROCK inhibitor Y27632 expressed lower levels of $\beta$-catenin, exhibited reduced nuclear localization of YAP/TAZ and exhibited a higher propensity for corneal differentiation compared with cells maintained in vehicle (Supplementary Fig. 7a-e).

Further evidence of a link between mechanotransduction and $\beta$-catenin signalling was provided by induction of TCF-luciferase activity in reporter cells following expression of a constitutively active ROCK2 kinase domain (Supplementary Fig. $7 \mathrm{f}-\mathrm{h}$ ), indicating that forced activation of the mechanotransduction cascade does indeed activate $\beta$-catenin signalling.

Collectively, these data support the hypothesis that activation of mechanotransduction is sufficient to promote elevated $\beta$-catenin activity in corneal epithelial cells and, in addition, suggest that this is a mechanism that is conserved across species.

\section{Modulation of mechanotransduction cascades regulates cell fate on the ocular surface}

To definitively establish that chronic inflammation promotes CSCM through mechanotransduction, we tested whether manipulation of the mechanotransduction cascade affects cell fate on the ocular surface in vivo. In the first instance, we determined whether inhibition of mechanotransduction could restore corneal differentiation in chronically inflamed Notch $^{\Delta}$ corneas by using small-molecule inhibitors of FAK (PF562271) or ROCK (Y27632; Fig. 8a). Strikingly, mice treated with either inhibitor retained a predominantly $\mathrm{K}_{12}{ }^{+} \mathrm{K} 1^{-}$ $\mathrm{CE}$, despite the presence of a chronically inflamed stroma (Fig. 8b-e), thus supporting the hypothesis that chronic inflammation elicits CSCM through activation of mechanotransduction.

To test the hypothesis further, we performed the reciprocal experiment by ablating YAP/TAZ from the CE, thus removing cytoplasmic YAP/TAZ and recapitulating the effect of mechanotransduction. Thus,

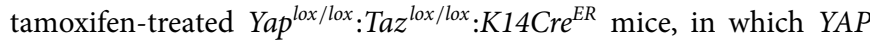
and $T A Z$ are ablated in the $\mathrm{CE}\left(Y A P^{\triangle}: T A Z^{\Delta}\right)$, were subjected to a single corneal injury and analysed 7 days later (Fig. 8f). Strikingly, the ocular surface of $Y A P^{\triangle}: T A Z^{\triangle}$ corneas exhibited overt epidermal differentiation, particularly in peripheral regions (Fig. 8g), which correlated with loss of YAP/TAZ (Fig. 8h) and increased $\beta$-catenin expression (Fig. 8i). As expected, WT controls maintained corneal identity throughout the CE (Fig. 8g-i).

Collectively, these data provide functional evidence that chronic inflammation induces CSCM by activating the mechanotransduction cascade.

\section{DISCUSSION}

In this study we have demonstrated that a central mechanism by which chronic inflammation can promote aberrant cell fate is through mechanotransduction (Fig. 8j). The data presented support a model whereby exposure of the corneal stroma to chronic inflammation results in the induction of excessive ECM deposition, which subsequently promotes epidermal differentiation in the regenerating epithelium through mechanical induction of $\beta$-catenin signalling. 
a

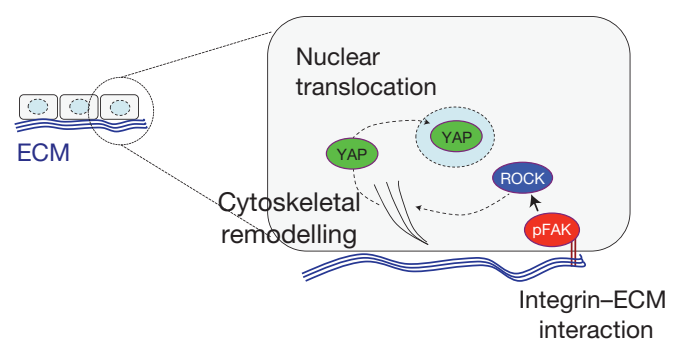

C

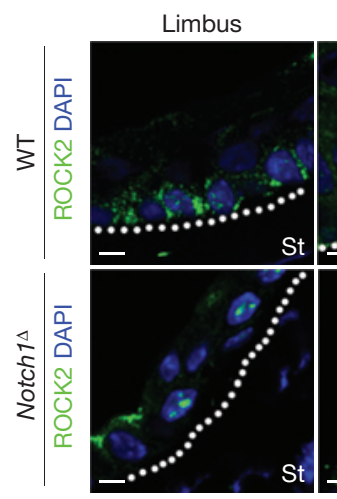

e

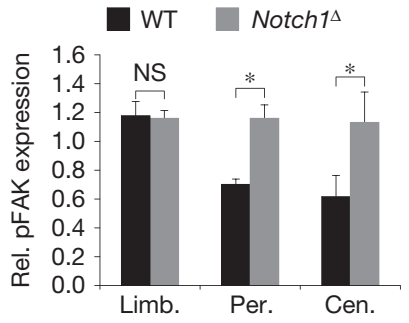

Peripheral

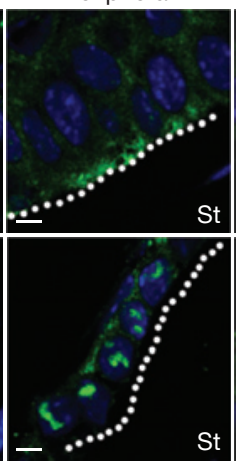

Central

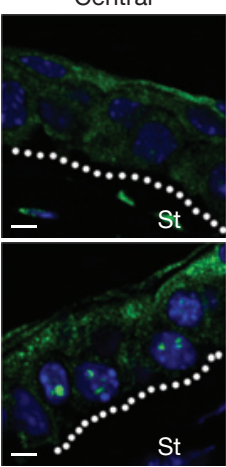

b

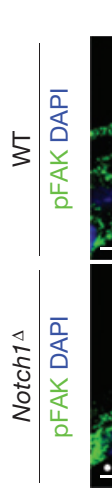

d
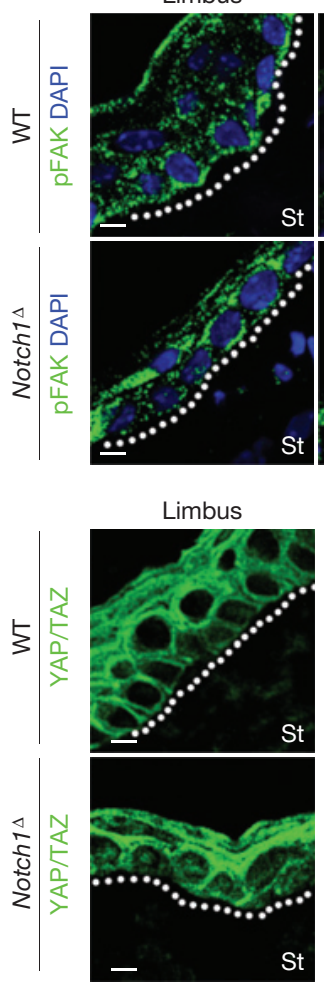

st

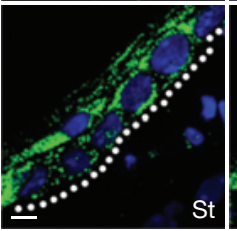

Limbus

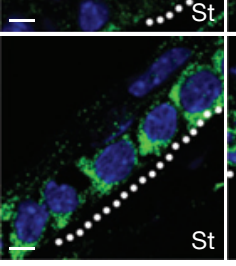

Peripheral
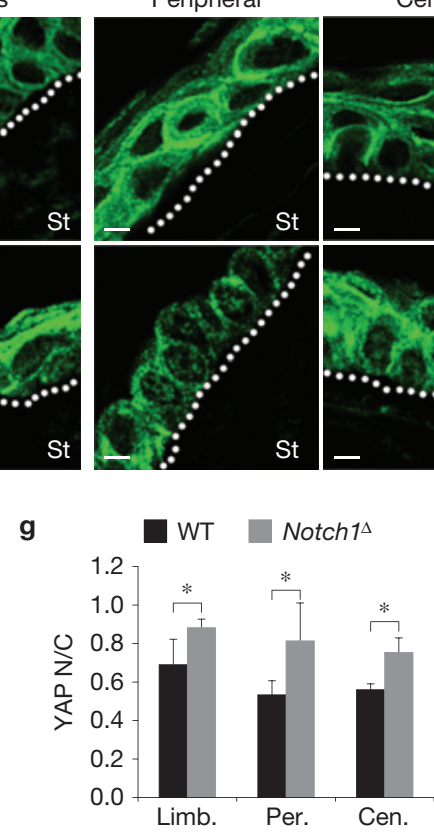

Central

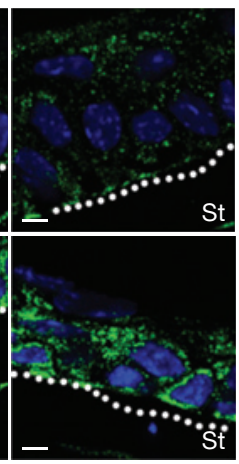

Centra

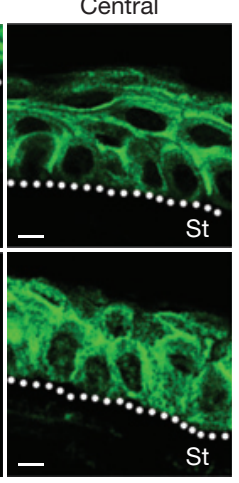

f

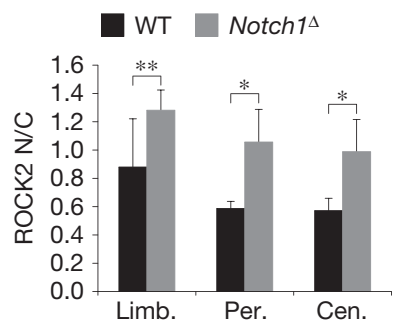

Values for expression levels are relative values normalized to the expression level in the conjunctiva of each sample, determined by mean fluorescence intensity. Black bars, WT (Notch $1^{\text {lox/lox }}$ ); grey bars, Notch $1^{\Delta}(n=6$ corneas for each genotype over three independent experiments). (f,g) Quantification of nuclear/cytoplasmic ratio (N/C) of ROCK2 (f) and YAP/TAZ (g) in the limbus, peripheral cornea and central cornea $24 \mathrm{~h}$ after the second injury. $n=6$ corneas for each genotype over three independent experiments. St, stroma; NS, not significant. ${ }^{*} P<0.01,{ }^{* *} P<0.05$ (unpaired, two-tailed $t$-tests). Error bars represent standard deviation. Scale bars represent $5 \mu \mathrm{m}$. White dotted lines indicate the boundary between the stroma and epithelium.
The mechanisms underlying ECM deposition in this model remain to be fully elucidated, although the expression of matrix components is confined to non-haematopoietic stromal cells, thus suggesting that the changes in the ECM occur owing to a fibrotic-like process. A variety of immune cells, including macrophages ${ }^{50}$, neutrophils ${ }^{51}$ and $\mathrm{T}$ cells ${ }^{52}$, are linked to the induction of fibrosis through the secretion of cytokines such as TGF- $\beta$ (refs 53,54) and IL-13 (refs 22,55), which subsequently induce excessive ECM deposition by $\alpha-\mathrm{SMA}^{+}$ myofibroblasts ${ }^{56-58}$. It will thus be interesting to determine whether similar cellular and molecular mechanisms are involved in the stromal remodelling observed in the chronically inflamed cornea. The profiles of inflammatory cell types in the corneal stroma of both WT and
Notch $1^{\Delta}$ mice are similar following injury and consist predominantly

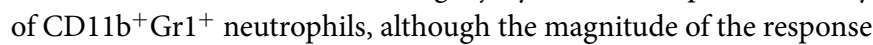
is elevated in $\operatorname{Notch}^{\Delta}{ }^{\Delta}$ mice. However, the prolonged duration of the inflammatory response in Notch1 mutants seems to be critical for the induction of stromal remodelling and resulting metaplasia, as both of these effects occur following repeated cycles of injury and repair. Experiments in which specific inflammatory cell types and cytokines can be efficiently depleted will enable delineation of the specific cellular and molecular mediators involved and may identify promising targets for therapeutic intervention in ocular surface disorders.

The induction of CSCM in the model described here is initially induced in the limbus and peripheral corneal epithelium, locations 
a
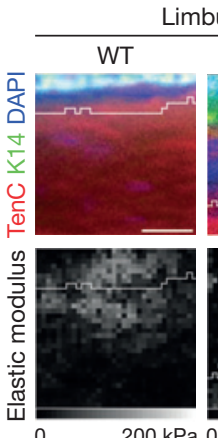

mbus
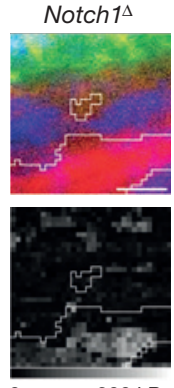

Peripheral cornea
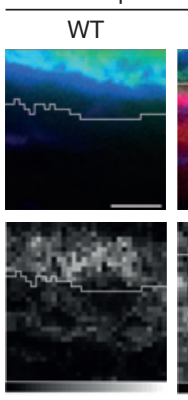

Notch $1^{\Delta}$
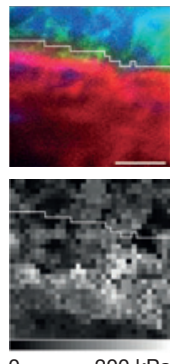
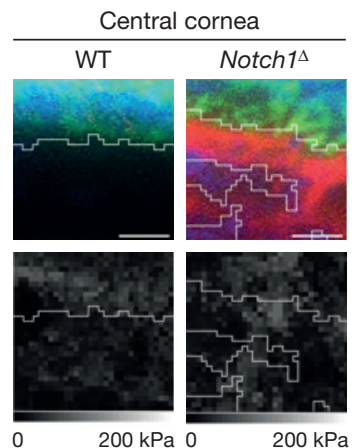

b

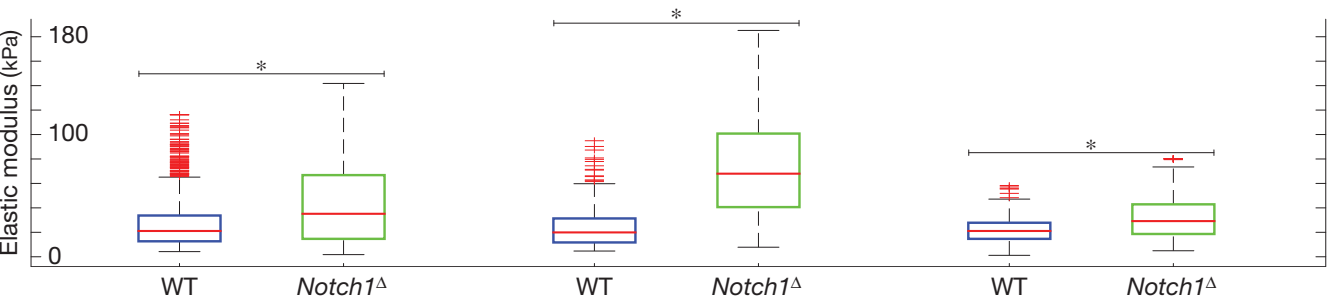

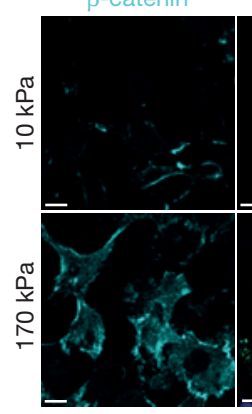

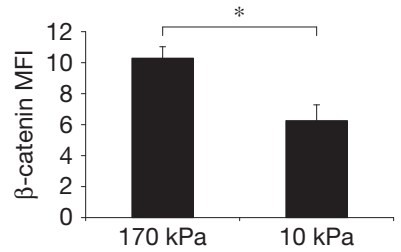

K12 K1 DAPI K12 K1 $\beta$-cat DAPI
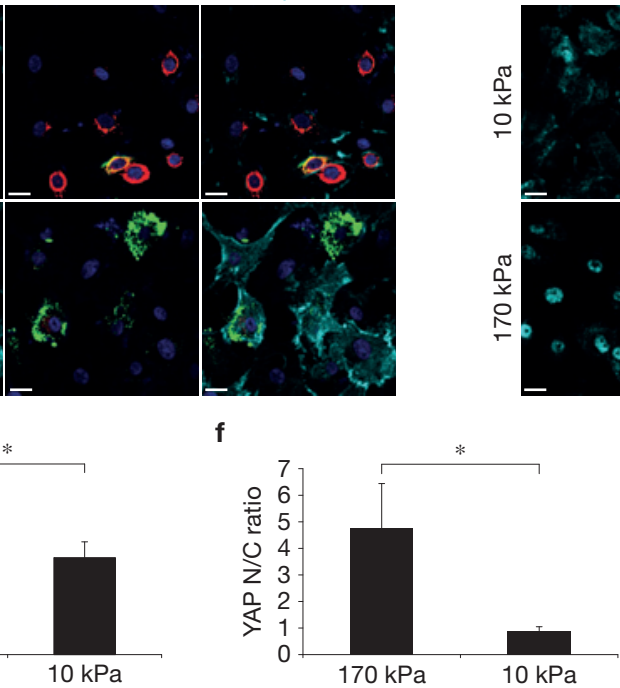

d

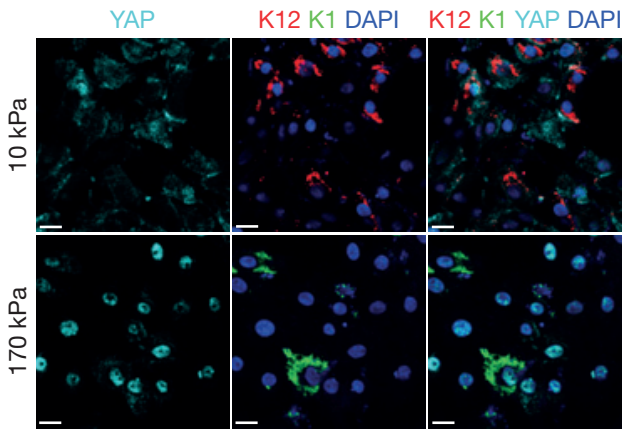

g

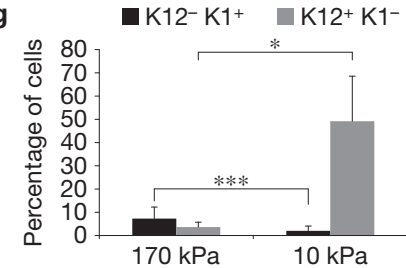

Figure $7 \mathrm{CSCM}$ is associated with increased tissue stiffness and mechanical stimuli. (a) Immunofluorescence for $\mathrm{K} 14$ and tenascin C (upper panels) and corresponding atomic force microscopy (AFM) nanomechanical property measurement (lower panels) of limbus, peripheral cornea and central cornea after repeated injury. The elastic modulus was determined using AFM forcevolume mode. Data are representative of 4 corneas per genotype over 4 independent experiments. (b) Quantification of the elastic modulus (kPa) of stromal tissue from the limbus (left), peripheral cornea (middle) and central cornea (right) of the regions shown in a. Red lines in each box represent the median elastic modulus value of 256 force-volume measurements in the regions shown in a. Red lines in each whisker represent outliers. Boxes represent the middle $50 \%$ of the data. (c,d) Immunofluorescence for $\beta$-catenin, $\mathrm{K} 12$ and $\mathrm{K} 1$ (c) or YAP/TAZ, K12 and $\mathrm{K} 1$ (d) on PCESCs cultured on soft (upper panels) or stiff (lower panels) substrates. Data are representative of 6 individual cultures over 2 independent experiments. (e-g) Quantification of $\beta$-catenin expression (MFI, mean fluorescence intensity) (e), YAP/TAZ nuclear/cytoplasmic ratio (N/C) (f) and the proportion of $\mathrm{K} 12^{+} \mathrm{K} 1^{-}$and $\mathrm{K} 12^{-} \mathrm{K} 1^{+}$cells (g) in PCESCs cultured on soft or stiff substrates ( $n=6$ for each condition over 2 independent experiments, where one replicate represents quantification from a single culture). For e, $\beta$-catenin expression is determined by mean fluorescence intensity. Scale bars in a represent $15 \mu \mathrm{m}$; scale bars in $\mathbf{c}$ and $\mathbf{d}$ represent $20 \mu \mathrm{m} .{ }^{*} P<0.01,{ }^{* * *} P<0.1$ (unpaired, two-tailed $t$-tests). Error bars represent standard deviation $(\mathbf{e}-\mathbf{g})$ or maximal values $/ 1.5 \times$ interquartile range (b). that contain significant numbers of activated stem/progenitor cells following injury. In addition, it is notable that although there is a significant overlap between elevated $\beta$-catenin expression and CSCM, not all $\beta$-catenin ${ }^{\text {hi }}$ cells undergo epidermal fate conversion. This therefore suggests that only a proportion of epithelial cells on the anterior ocular surface are permissive to $\beta$-catenin-induced fate switching. Given that the induction of CSCM coincides temporally and spatially with the activation of stem/progenitor cells in the limbus and peripheral cornea, it is tempting to speculate that these cells represent uncommitted progenitor cells that retain the potency to form epidermis in response to elevated $\beta$-catenin. To formally prove this hypothesis, phenotypic markers that precisely identify stem and progenitor cells in the corneal epithelium are required to definitively establish the cellular target of $\beta$-catenin-induced CSCM. 
a

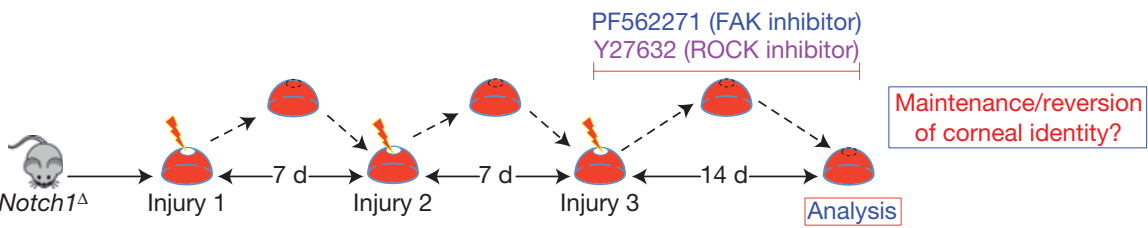

b

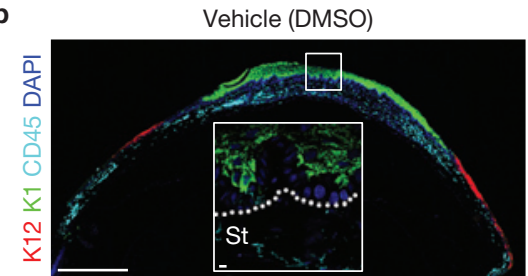

d

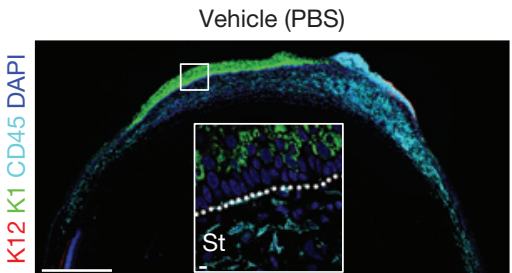

PF562271 (FAK inhibitor)

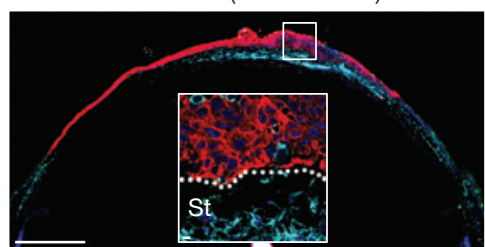

Y27632 (ROCK inhibitor)

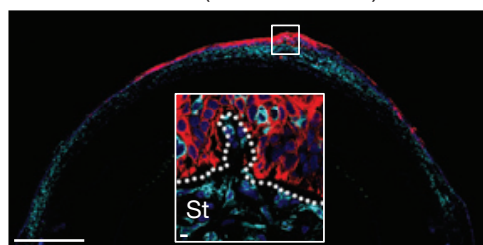

c Epidermal conversion

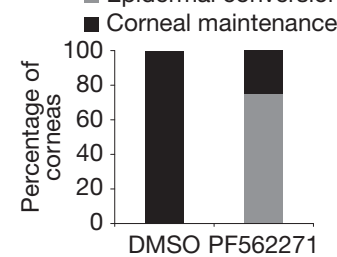

e

Epidermal conversion - Corneal maintenance

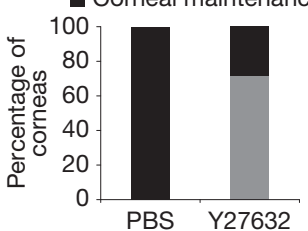

f Yaplox/lox:Tazlox/lox: K14Cre ${ }^{E R}$

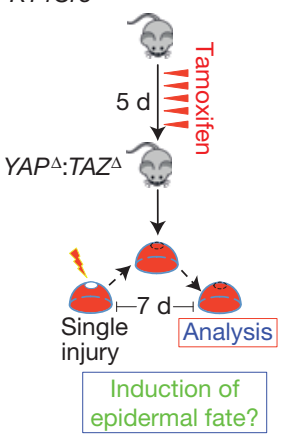

g
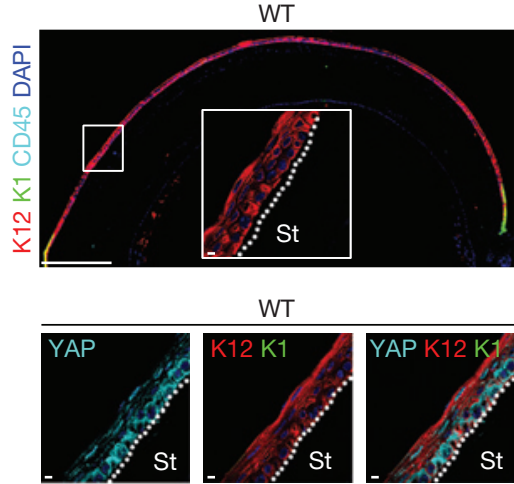

WT

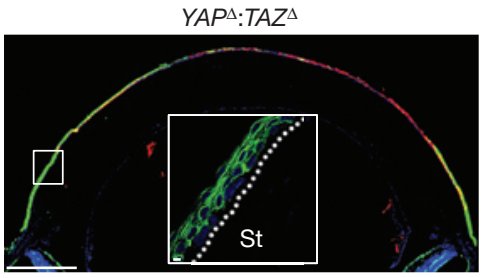

$Y A P^{\triangle}: T A Z^{\triangle}$

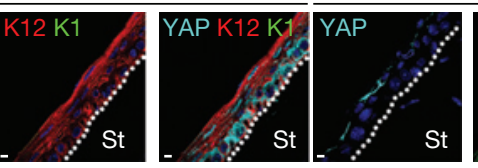

$Y A P^{\triangle}: T A Z^{\Delta}$

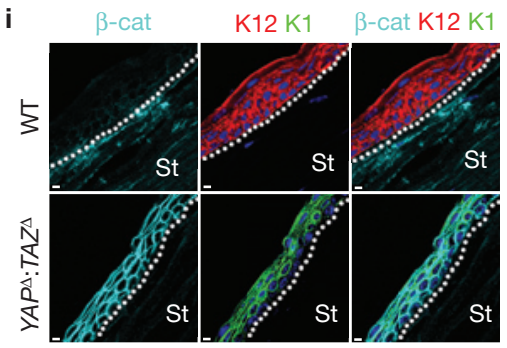

i

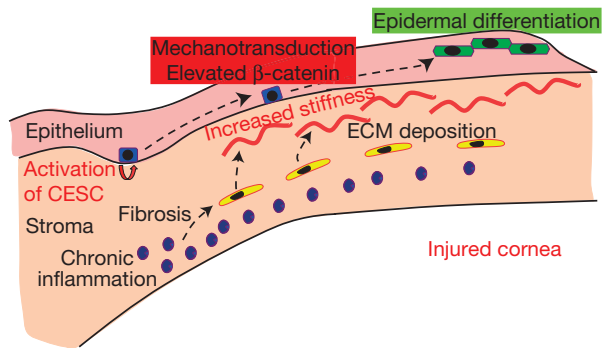

Figure 8 Manipulation of mechanotransduction affects corneal cell fate. (a) Schematic depiction of the experimental strategy used to determine whether inhibition of mechanotransduction prevents CSCM. (b) Immunofluorescent staining for $\mathrm{K} 12, \mathrm{~K} 1$ and $\mathrm{CD} 45$ on Notch $1^{\Delta}$ corneas treated with vehicle (dimethylsulphoxide, DMSO) or the FAK inhibitor PF562271 during the procedure outlined in $\mathbf{a}$. The images are representative of 8 corneas per treatment over four independent experiments. Large panels are low-magnification tiled images. Insets show high-magnification images of the indicated regions. (c) Quantification of the proportion of Notch $1^{\Delta}$ corneas exhibiting corneal or epidermal differentiation during wound closure following treatment with vehicle or PF562271 ( $n=8$ corneas for each treatment over four independent experiments). (d) Immunofluorescent staining for $\mathrm{K} 12, \mathrm{~K} 1$ and $\mathrm{CD} 45$ on Notch $1^{\Delta}$ corneas treated with vehicle (PBS) or the ROCK inhibitor Y27632 after the procedure outlined in a. The images are representative of 10 corneas per treatment over four independent experiments. Large panels are low-magnification tiled images. Insets show high-magnification images of the indicated regions. (e) Quantification of the proportion of Notch $1^{\Delta}$ corneas exhibiting corneal or epidermal differentiation during wound closure following treatment with vehicle or Y27632 ( $n=10$ corneas for each treatment over four independent experiments). (f) Schematic depiction of the experimental strategy used to determine whether ablation of YAP/TAZ is sufficient to induce CSCM during repair. (g) Immunofluorescent staining for $\mathrm{K} 12, \mathrm{~K} 1$ and $\mathrm{CD} 45$ on WT $\left(\mathrm{Yap}^{+/+}: \mathrm{TaZ}^{+/+}: \mathrm{K} 14 \mathrm{Cre} \mathrm{CR}^{E R}\right)$ and $Y A P^{\triangle}: T A Z^{\Delta}$ corneas after the procedure outlined in $\mathbf{f}$. The images are representative of 6 corneas for WT and 7 corneas for $Y A P^{\triangle}: T A Z^{\triangle}$ isolated over two independent experiments. Large panels are low-magnification tiled images. Insets show high-magnification images of the indicated regions. (h,i) Immunofluorescent staining for YAP/TAZ, $\mathrm{K} 12$ and $\mathrm{K} 1$ (h) and $\beta$-catenin, $\mathrm{K} 12$ and $\mathrm{K} 1$ (i) in WT and $Y A P^{\triangle}: T A Z^{\Delta}$ corneas as indicated. The images are representative of 6 corneas for WT and 7 corneas for $Y A P^{\triangle}: T A Z^{\triangle}$ isolated over two independent experiments. (j) Model depicting how chronic inflammation imposes aberrant cell fate on corneal epithelial stem cells through increased tissue stiffness and mechanotransduction. Scale bars represent $500 \mu \mathrm{m}$ on tiled images and $5 \mu \mathrm{m}$ on all other images. St, stroma. White dotted lines indicate the boundary between the stroma and epithelium. 
Finally, although this study demonstrates that mechanotransduction is a key mechanism by which chronic inflammation can impose aberrant cell fate, the mechanisms by which inflammation influences other aspects of stem/progenitor cell function are likely to be multifaceted. Notably, although inhibition of mechanotransduction and/or $\beta$-catenin signalling restores corneal identity in a chronic inflammatory environment, the epithelium remains hyperplastic. Thus, inflammation is likely to effect traits such as cell fate and proliferation through distinct mechanisms.

In summary, our findings demonstrate that chronic inflammation can promote aberrant cell fate through mechanotransduction and thus reveal an important mechanism by which aberrant inflammatory responses can promote the development of disease.

\section{METHODS}

Methods and any associated references are available in the online version of the paper.

Note: Supplementary Information is available in the online version of the paper

\section{ACKNOWLEDGEMENTS}

This work was supported in part by OptiStem, the Swiss National Science Foundation, the Swiss Cancer League, the Marie Curie Foundation and EuroSystem. P.D.O. and G.E.F. acknowledge financial support from the Swiss National Science Foundation under award number 205321_134786 and 205320_152675 and European Union FP7/2007-2013 ERC under Grant Agreement No. 307338NaMic. Work performed in the laboratory of S.P. was supported by grants from AIRC (5x1000 and PI) and the ERC. We thank R. Kemler (Max Planck Institute of Immunobiology and Epigenetics, Freiburg, Germany) for providing the conditional $\beta$-catenin mice, M. Taketo (Centre for Frontier Medicine, Kyoto University, Japan) for the $\mathrm{Ctnnb}^{\Delta}{ }^{\Delta} \mathrm{Ex} 3$ mice and P. Chambon and D. Metzger (Institute of Genetics and Molecular and Cellular Biology, Strasbourg, France) for the $\mathrm{K} 5 \mathrm{Cre}{ }^{\mathrm{ERT}}$ and $\mathrm{K} 14 \mathrm{Cre}^{\mathrm{ERT} T}$ mice. We thank J. Huelsken (Swiss Institute for Experimental Cancer Research, EPFL, Lausanne, Switzerland) for providing the TCF-luciferase reporter cells and the anti-periostin antibody. We would like to thank A. Radenovic, J. Artacho, J. Sordet-Dessimoz and M. Garcia for technical assistance with microscopy, histology and flow cytometry. We would like to thank G. Ferrand for guidance and advice concerning animal experiments.

\section{AUTHOR CONTRIBUTIONS}

C.S.N. designed and performed experiments, analysed data and wrote the manuscript. P.D.O. performed nanomechanical measurement experiments and analysed data. L.A. performed experiments and analysed data. S.H. performed experiments and analysed data. E.F.W., G.E.F., M.P.L., Y.B. and S.P. analysed data and provided conceptual and experimental guidance throughout the study. F.R. conceived the study and analysed data.

\section{COMPETING FINANCIAL INTERESTS}

The authors declare no competing financial interests.

Published online at http://dx.doi.org/10.1038/ncb3290

Reprints and permissions information is available online at www.nature.com/reprints

1. Arwert, E. N., Hoste, E. \& Watt, F. M. Epithelial stem cells, wound healing and cancer. Nat. Rev. Cancer 12, 170-180 (2012).

2. Grivennikov, S. I., Greten, F. R. \& Karin, M. Immunity, inflammation, and cancer. Cell 140, 883-899 (2010).

3. Herfs, M., Hubert, P. \& Delvenne, P. Epithelial metaplasia: adult stem cell reprogramming and (pre)neoplastic transformation mediated by inflammation? Trends Mol. Med. 15, 245-253 (2009)

4. Karin, M. Nuclear factor-KB in cancer development and progression. Nature 441, 431-436 (2006)

5. Tu, S. et al. Overexpression of interleukin-1 $\beta$ induces gastric inflammation and cancer and mobilizes myeloid-derived suppressor cells in mice. Cancer Cell 14, 408-419 (2008)

6. Wu, S. et al. A human colonic commensal promotes colon tumorigenesis via activation of T helper type 17 T cell responses. Nat. Med. 15, 1016-1022 (2009).

7. Quante, M. et al. Bile acid and inflammation activate gastric cardia stem cells in a mouse model of Barrett-like metaplasia. Cancer Cell 21, 36-51 (2012).
8. Barker, N. et al. Crypt stem cells as the cells-of-origin of intestinal cancer. Nature 457, 608-611 (2009).

9. Driessens, G., Beck, B., Caauwe, A., Simons, B. D. \& Blanpain, C. Defining the mode of tumour growth by clonal analysis. Nature 488, 527-530 (2012).

10. Shenoy, A. K. et al. Transition from colitis to cancer: high Wnt activity sustains the tumor-initiating potential of colon cancer stem cell precursors. Cancer Res. $\mathbf{7 2}$, 5091-5100 (2012)

11. Boulter, L. et al. Macrophage-derived Wnt opposes Notch signaling to specify hepatic progenitor cell fate in chronic liver disease. Nat. Med. 18, 572-579 (2012).

12. Taniguchi, K. et al. A gp130-Src-YAP module links inflammation to epithelial regeneration. Nature 519, 57-62 (2015).

13. Di Piazza, M., Nowell, C. S., Koch, U., Durham, A. D. \& Radtke, F. Loss of cutaneous TSLP-dependent immune responses skews the balance of inflammation from tumor protective to tumor promoting. Cancer Cell 22, 479-493 (2012).

14. Aragona, M. et al. A mechanical checkpoint controls multicellular growth through YAP/TAZ regulation by actin-processing factors. Cell 154, 1047-1059 (2013).

15. Samuel, M. S. \& Olson, M. F. Actomyosin contractililty: force power drives tumor growth. Cell Cycle 10, 3409-3410 (2011).

16. Humphrey, J. D., Dufresne, E. R. \& Schwartz, M. A. Mechanotransduction and extracellular matrix homeostasis. Nat. Rev. Mol. Cell Biol. 15, 802-812 (2014).

17. Paszek, M. J. et al. Tensional homeostasis and the malignant phenotype. Cancer Cell 8, 241-254 (2005).

18. Levental, K. R. et al. Matrix crosslinking forces tumor progression by enhancing integrin signaling. Cell 139, 891-906 (2009).

19. Bonnans, C., Chou, J. \& Werb, Z. Remodelling the extracellular matrix in development and disease. Nat. Rev. Mol. Cell Biol. 15, 786-801 (2014).

20. McHedlidze, T. et al. Interleukin-33-dependent innate lymphoid cells mediate hepatic fibrosis. Immunity 39, 357-371 (2013).

21. Aceves, S. S. \& Ackerman, S. J. Relationships between eosinophilic inflammation, tissue remodeling, and fibrosis in eosinophilic esophagitis. Immunol. Allergy Clin. North Am. 29, 197-211 (2009).

22. Zhu, Z. et al. Pulmonary expression of interleukin-13 causes inflammation, mucus hypersecretion, subepithelial fibrosis, physiologic abnormalities, and eotaxin production. J. Clin. Invest. 103, 779-788 (1999).

23. Daniels, J. T., Dart, J. K., Tuft, S. J. \& Khaw, P. T. Corneal stem cells in review. Wound Repair Regen. 9, 483-494 (2001).

24. Daniels, J. T. et al. Limbal epithelial stem cell therapy. Expert Opin. Biol. Ther. 7, $1-3$ (2007).

25. Majo, F., Rochat, A., Nicolas, M., Jaoude, G. A. \& Barrandon, Y. Oligopotent stem cells are distributed throughout the mammalian ocular surface. Nature $\mathbf{4 5 6}$, 250-254 (2008).

26. Liang, L., Sheha, H., Li, J. \& Tseng, S. C. Limbal stem cell transplantation: new progresses and challenges. Eye 23, 1946-1953 (2009).

27. Samson, C. M., Nduaguba, C., Baltatzis, S. \& Foster, C. S. Limbal stem cell transplantation in chronic inflammatory eye disease. Ophthalmology 109, 862-868 (2002).

28. McNamara, N. A., Gallup, M. \& Porco, T. C. Establishing PAX6 as a biomarker to detect early loss of ocular phenotype in human patients with Sjogren's syndrome. Invest. Ophthalmol. Vis. Sci. 55, 7079-7084 (2014).

29. Chen, Y. T. et al. Immune profile of squamous metaplasia development in autoimmune regulator-deficient dry eye. Mol. Vis. 15, 563-576 (2009).

30. Chen, Y. T. et al. Interleukin-1 receptor mediates the interplay between $\mathrm{CD}^{+} \mathrm{T}$ cells and ocular resident cells to promote keratinizing squamous metaplasia in Sjogren's syndrome. Lab. Invest. 92, 556-570 (2012).

31. Vauclair, S. et al. Corneal epithelial cell fate is maintained during repair by Notch1 signaling via the regulation of vitamin A metabolism. Dev. Cell 13, 242-253 (2007).

32. Demehri, S. et al. Notch-deficient skin induces a lethal systemic Blymphoproliferative disorder by secreting TSLP, a sentinel for epidermal integrity. PLoS Biol. 6, e123 (2008).

33. Dumortier, A. et al. Atopic dermatitis-like disease and associated lethal myeloproliferative disorder arise from loss of Notch signaling in the murine skin. PLOS ONE 5, e9258 (2010).

34. Guinea-Viniegra, J. et al. Differentiation-induced skin cancer suppression by FOS, p53, and TACE/ADAM17. J. Clin. Invest. 122, 2898-2910 (2012).

35. Murthy, A. et al. Notch activation by the metalloproteinase ADAM17 regulates myeloproliferation and atopic barrier immunity by suppressing epithelial cytokine synthesis. Immunity 36, 105-119 (2012).

36. Schonthaler, H. B., Guinea-Viniegra, J. \& Wagner, E. F. Targeting inflammation by modulating the Jun/AP-1 pathway. Ann. Rheum. Dis. 70, i109-i112 (2011).

37. Eckert, R. L. et al. AP1 transcription factors in epidermal differentiation and skin cancer. J. Skin Cancer 2013, 537028 (2013).

38. Wurm, S. et al. Terminal epidermal differentiation is regulated by the interaction of Fra-2/AP-1 with Ezh2 and ERK1/2. Genes Dev. 29, 144-156 (2015).

39. Chappaz, S., Flueck, L., Farr, A. G., Rolink, A. G. \& Finke, D. Increased TSLP availability restores T- and B-cell compartments in adult IL-7 deficient mice. Blood 110, 3862-3870 (2007).

40. Omori-Miyake, M. \& Ziegler, S. F. Mouse models of allergic diseases: TSLP and its functional roles. Allergol. Int. 61, 27-34 (2012).

41. Kuraguchi, M. et al. Adenomatous polyposis coli (APC) is required for normal development of skin and thymus. PLoS Genet. 2, e146 (2006). 
42. Miyoshi, K. et al. Activation of $\beta$-catenin signaling in differentiated mammary secretory cells induces transdifferentiation into epidermis and squamous metaplasias. Proc. Natl Acad. Sci. USA 99, 219-224 (2002).

43. Mukhopadhyay, M. et al. Dkk2 plays an essential role in the corneal fate of the ocular surface epithelium. Development 133, 2149-2154 (2006).

44. Harada, N. et al. Intestinal polyposis in mice with a dominant stable mutation of the $\beta$-catenin gene. EMBO J. 18, 5931-5942 (1999)

45. Dupont, S. et al. Role of YAP/TAZ in mechanotransduction. Nature 474, 179-183 (2011).

46. Azzolin, L. et al. YAP/TAZ incorporation in the $\beta$-catenin destruction complex orchestrates the Wnt response. Cell 158, 157-170 (2014).

47. Iredale, J. P. Models of liver fibrosis: exploring the dynamic nature of inflammation and repair in a solid organ. J. Clin. Invest. 117, 539-548 (2007).

48. Carey, W. A., Taylor, G. D., Dean, W. B. \& Bristow, J. D. Tenascin-C deficiency attenuates TGF-ss-mediated fibrosis following murine lung injury. Am. J. Physiol. Lung. Cell Mol. Physiol. 299, L785-L793 (2010).

49. Naik, P. K. et al. Periostin promotes fibrosis and predicts progression in patients with idiopathic pulmonary fibrosis. Am. J. Physiol. Lung. Cell Mol. Physiol. 303, L1046-L1056 (2012).

50. Lawrance, I. C. et al. Cellular and molecular mediators of intestinal fibrosis. J. Crohn's Colitis http://dx.doi.org/10.1016/j.crohns.2014.09.008 (2015).
51. Sziksz, E. et al. Fibrosis related inflammatory mediators: role of the IL-10 cytokine family. Mediat. Inflamm. 2015, 764641 (2015).

52. Kullberg, M. C. et al. TGF- $\beta 1$ production by $\mathrm{CD} 4^{+} \mathrm{CD} 25^{+}$regulatory $\mathrm{T}$ cells is not essential for suppression of intestinal inflammation. Eur. J. Immunol. 35, 2886-2895 (2005).

53. Kenyon, N. J., Ward, R. W., McGrew, G. \& Last, J. A. TGF- $\beta 1$ causes airway fibrosis and increased collagen I and III mRNA in mice. Thorax 58, 772-777 (2003).

54. Wang, B. et al. Suppression of microRNA-29 expression by TGF- $\beta 1$ promotes collagen expression and renal fibrosis. J. Am. Soc. Nephrol. 23, 252-265 (2012).

55. Fulkerson, P. C., Fischetti, C. A. \& Rothenberg, M. E. Eosinophils and CCR3 regulate interleukin-13 transgene-induced pulmonary remodeling. Am. J. Pathol. 169, 2117-2126 (2006)

56. Eyden, B. The myofibroblast: phenotypic characterization as a prerequisite to understanding its functions in translational medicine. J. Cell Mol. Med. 12 , 22-37 (2008).

57. De Wever, O., Demetter, P., Mareel, M. \& Bracke, M. Stromal myofibroblasts are drivers of invasive cancer growth. Int. J. Cancer 123, 2229-2238 (2008).

58. Lin, S. L., Kisseleva, T., Brenner, D. A. \& Duffield, J. S. Pericytes and perivascular fibroblasts are the primary source of collagen-producing cells in obstructive fibrosis of the kidney. Am. J. Pathol. 173, 1617-1627 (2008). 


\section{METHODS}

Ethics statement. All animal work was conducted in accordance with Swiss national guidelines. All mice were kept in the animal facility under EPFL animal care regulations. They were housed in individual cages at $23^{\circ} \mathrm{C} \pm 1{ }^{\circ} \mathrm{C}$ with a $12 \mathrm{~h}$ light/dark cycle. All animals were supplied with food and water ad libitum. This study has been reviewed and approved by the Service Veterinaire Cantonal of Etat de Vaud.

Mice. Notch $1^{\text {lox/lox }}, C t n n b 1^{\text {lox } / l o x}$ and $K 5 C r e^{E R T}$ mice were as previously described ${ }^{59-61}$ and were maintained on a C57BL/6 background. $c j u n^{\text {lox } / l o x}$ mice were as previously

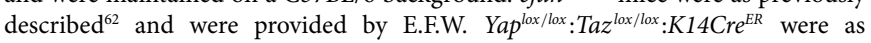
previously described ${ }^{5}$ provided by S.P. These mice were backcrossed onto the C57BL/6 background. Ctnnb1 $1^{\text {lox (ex3) }}$ and K14TSLPTg mice were as previously described $^{6,7}$ and were maintained on an sv129 background. The conditional

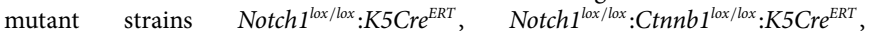
Notch $1^{l o x} / / 0 x: c j u n^{l o x} /$ lox $: K 5 C r e^{E R T}$ and Ctnnb1 $1^{\operatorname{lox}(e x 3) / \operatorname{lox}(e \times 3)}$ were generated by performing the required inter-crosses and were maintained as homozygotes. For conditional ablation of YAP/TAZ, Yap ${ }^{l o x / l o x}: T a z^{l o x} / l o x: K 14 C r e^{E R}$ mice were administered with $1 \mathrm{mg}$ tamoxifen once a day for 3 days by intraperitoneal injection. For all other gene activation/inactivation experiments, tamoxifen dissolved in peanut oil was administered once a day for 5 consecutive days by intraperitoneal injection at a dose of $50 \mathrm{mg} \mathrm{kg}^{-1}$. For each experiment, all mice were age matched and were between 4 and 7 weeks of age. Male and female animals were used. All mouse experiments were non-randomized and non-blinded; a minimum of three animals were used for each experiment, allowing a minimum of six corneas to be analysed for each experiment. This number was deemed sufficiently appropriate to account for normal variation, as determined from previous studies. Numbers of animals for each experiment are described in the figure legends. No statistical method was used to predetermine sample size.

Statistical analysis. For statistical analysis, unpaired two-tailed $t$-tests were performed on normally distributed data sets with equal variance assumed. Unless otherwise stated, $n$ numbers for experiments stating statistical significance represent biological replicates. Sample size in all cases was a minimum of 6 independent biological replicates, according to availability of biological material.

Corneal wounding. Mice were anaesthetized by sub-cutaneous injection of ketamine $\left(100 \mathrm{mg} \mathrm{kg}^{-1}\right)$ and xylazine $\left(10 \mathrm{mg} \mathrm{kg}^{-1}\right)$. Anaesthetic collrium $(0.4 \%$ oxybuprocaine) was applied to the ocular surface to prevent corneal reflex.

To wound the ocular surface, a circular area $2 \mathrm{~mm}$ in diameter was marked on the central cornea by gentle application of a $2 \mathrm{~mm}$ biopsy punch. Removal of the epithelial tissue within this area was then achieved using a Demarres spatula (Moria Surgical) with a $45^{\circ}$ cutting angle. The efficiency of wounding was monitored by application of $0.5 \%$ solution of ophthalmic fluorescein (Novartis). Following corneal wounding, Viscotears ophthalmic gel (Novartis) was applied to the ocular surface.

On waking, mice were administered with $0.3 \mathrm{ml} 0.9 \% \mathrm{NaCl}$ solution (Braun) to aid recovery. Dafalgen analgesic was provided in the drinking water following corneal wounding at a concentration of $2 \mathrm{mg} \mathrm{ml}^{-1}$.

Administration of Tobradex anti-inflammatory gel. Tobradex gel or ophthalmic lubricating gel was administered topically to the ocular surface once a day throughout the corneal wounding procedure, starting from the day of the first corneal wound and ending 21 days after the third corneal wound.

Administration of PF562271 (FAK inhibitor) and Y27632 (ROCK inhibitor). For systemic administration, PF562271 (Selleckchem) was dissolved in 70\% DMSO/dH2O and injected i.p. at a dose of $30 \mathrm{mg} \mathrm{kg}^{-1}$ every other day for 14 days following the final corneal injury. Control mice were injected with 70\% DMSO vehicle alone. For topical application, a $3 \mu \mathrm{l}$ drop of $10 \mathrm{mM}$ PF562271 was applied to the ocular surface twice a day for 14 days following the final corneal injury in a $15 \%$ DMSO solution (diluted in $\mathrm{dH} 20$ ). Control mice were administered with $15 \%$ DMSO vehicle alone.

For systemic administration, Y27632 (Sigma) was dissolved in saline solution and injected i.p. at a dose of $30 \mathrm{mg} \mathrm{kg}^{-1}$ every other day for 14 days following the final corneal injury. Control mice were injected with saline vehicle alone.

For topical application, a $3 \mu \mathrm{l}$ drop of $10 \mathrm{mM}$ Y27632 diluted in saline was applied to the ocular surface twice a day for 14 days following the final corneal injury. Control mice were administered with saline vehicle alone.

Cell culture. Peripheral corneal epithelial cells were isolated from porcine corneas and cultivated on lethally irradiated 3T3-J2 feeder cells in 3:1 DMEM/F12 medium supplemented as previously described ${ }^{63}$.

For experiments analysing the effect of substrate stiffness, cells were plated on glass coverslips, standard tissue culture plastic, 15\% PEG or 2.5\% PEG hydrogels in the absence of 3T3-J2 feeders at a density of 50,000 cells cm$~^{-2}$. For experiments using the ROCK inhibitor Y27632, cells were seeded onto glass coverslips in the absence of 3T3-J2 feeders at a density of 50,000 cells $\mathrm{cm}^{-2}$. All substrates in feeder-free cultures were coated with recombinant human collagen I (AteloCell). Glass coverslips were treated with poly-L-lysine (Sigma) before collagen coating.

Hydrogel substrates were formed by crosslinking two branched PEG precursors of relative molecular mass 10,000, end-functionalized with either thiol (TH) or vinylsulphone (VS) groups (NOF Corporation). To adjust the stiffness the precursors were mixed at different mass to volume ratios. Fifty microlitres of the mixed hydrogel precursor solution were transferred to each well of a 12-well plate (Falcon). A hydrophobic PDMS stamp with spacers was used to produce homogeneous and thin gel layers. The partially crosslinked hydrogel substrate was subsequently functionalized with Collagen I (AteloCell) under pressure for $2 \mathrm{~h}$. The functionalized hydrogel layers were then washed with PBS and ultraviolet-sterilized.

Transient transfection of porcine corneal epithelial cells was performed using JetPEI transfection reagent (PolyPlus) according to the manufacturer's instructions.

TCF-Luc 293T cells were maintained in DMEM supplemented with 10\% FCS.

TCF-luciferase reporter assay. $293 \mathrm{~T}$ cells with a stably integrated luciferase cassette containing upstream TCF-binding sites were a kind gift from J. Huelsken. To assay TCF-luciferase activity in the presence or absence of the murine ROCK2 kinase domain, TCF-Luc $293 \mathrm{~T}$ cells were transiently co-transfected with $\mathrm{pR} 2 \mathrm{KD} / \mathrm{pRenilla}$ or $\mathrm{pEGFP/pRenilla} \mathrm{using} \mathrm{JetPEI} \mathrm{(PolyPlus)} \mathrm{transfection} \mathrm{reagent} \mathrm{according} \mathrm{to} \mathrm{the}$ manufacturer's instructions. Luciferase activity was subsequently analysed $36-48 \mathrm{~h}$ later using the Dual-Luciferase Reporter Assay System (Promega) and a Berthold Lumat Luminometer. Luciferase values were normalized to Renilla-luciferase values.

Preparation of corneal single-cell suspensions and flow cytometry. Whole corneal tissue was isolated from murine eyes, placed in ice-cold PBS and cut into small fragments using a scalpel blade. Corneal fragments were then digested in $2 \mathrm{mg} \mathrm{ml}^{-1}$ collagenase IV (Invitrogen)/PBS for $30-45 \mathrm{~min}$ at $37^{\circ} \mathrm{C}$ with gentle shaking. The resulting cell suspensions were then passed through a $70 \mu \mathrm{m}$ cell strainer into $1 \times \mathrm{HBSS} / 25 \mathrm{mM}$ HEPES $/ 2 \%$ newborn bovine calf serum (staining medium). Cells were subsequently washed twice with staining medium and then stained according to standard flow cytometry protocols with the following monoclonal antibody conjugates: anti-CD45-Pacific blue (clone 30-F11, Invitrogen), anti-CD11b-PE (clone M1/70, Biolegend), anti-Gr-1-Alexa 647 (clone RB6-8C5, EPFL Protein Core Facility), anti-CD11c-APC-eFluor 780 (clone N418, eBiosciences), anti-B220-PECy7 (clone RA3-6B2, eBiosciences), antiTCRB-APC-eFluor 780 (clone H57-597, eBiosciences), anti-CD4-PE (clone GK1.5, EPFL Protein Core Facility), anti-CD8-Alexa Fluor 647 (clone YTS169.4, EPFL Protein Core Facility), anti-F4/80-biotin (clone BM8, eBiosciences), StreptavidinFITC (eBiosciences). Before analysis, propidium iodide (Sigma) was added to cell suspensions at a final concentration of $5 \mu \mathrm{g} \mathrm{ml}^{-1}$ to enable dead cell exclusion. Data acquisition was performed using a Cyan flow cytometer (Dako) and analysed using FlowJo software (Tree Star).

Molecular cloning and generation of the $\mathrm{pR} 2 \mathrm{KD}$ plasmid. cDNA synthesis was performed using the Quantitect Reverse Transcription kit (Qiagen) on RNA isolated from mouse corneal tissue. The region of cDNA encompassing the first 417 amino acids of the amino-terminal kinase domain was subsequently PCR cloned in two fragments; a $641 \mathrm{bp} 5^{\prime}$ fragment starting $12 \mathrm{bp}$ upstream of the initiating ATG and a $659 \mathrm{bp} \mathrm{3}$ ' fragment starting $591 \mathrm{bp}$ downstream of the initiating ATG. A $38 \mathrm{bp}$ overlapping region was present in each fragment. The primers used to generate each fragment were as follows: $5^{\prime}$ fragment: Fwd $5^{\prime}$-AATTGTCCAGA ATTCGGCGAAGCGGCATGAGC-3'; Rev 5'-TAAGCCCATGGAGTGTA TTGC-3'; 3' fragment: Fwd 5'-GTAGTGCTTGCACTGGATGC-3'; Rev 5'CTACTACTATCTAAAGTAGGTAAATCCTATAAAAGGCAGC-3'.

The above primers enabled the incorporation of a $5^{\prime}$ Sall site (bold underlined) and $\times 33^{\prime}$ STOP codons (bold double underlined) in the $5^{\prime}$ and $3^{\prime}$ fragments respectively.

Cycling conditions were as follows: $5^{\prime}$ fragment $\times 35$ cycles of $94{ }^{\circ} \mathrm{C}, 30 \mathrm{s:}: 62^{\circ} \mathrm{C}$ $30 \mathrm{s:} 72^{\circ} \mathrm{C} 30 \mathrm{~s} ; 3^{\prime}$ fragment $\times 35$ cycles of $94^{\circ} \mathrm{C}, 30 \mathrm{~s}: 60^{\circ} \mathrm{C} 30 \mathrm{~s}: 720 \mathrm{C} 30 \mathrm{~s}$.

PCR was performed using Q5 DNA polymerase (NEB) according to the manufacturer's instructions, with the addition of DMSO to a final concentration of $5 \%$. Amplified fragments were cloned using the TOPO TA cloning system (Invitrogen).

The $5^{\prime}$ and $3^{\prime}$ fragments were inserted into an expression vector (pI-EGFP2) downstream of a CMV promoter/enhancer and upstream of an IRESEGFP cassette through a three-way ligation using SalI, NcoI (present in the overlapping region of each fragment) and Not-I restriction sites, resulting in the generation of PR2KD.

Tissue processing for histology. Fresh frozen and paraffin-embedded tissues were used for histological analysis. For fresh frozen tissue, whole eyes were embedded in OCT compound (TissueTek) using standard protocols. Eight-micrometre-thick sections were cut using a Leica CM1850 cryostat. Cryosections were stored at $-80^{\circ} \mathrm{C}$. 
For paraffin-embedded tissue, whole eyes were fixed in 4\% PFA overnight at $4{ }^{\circ} \mathrm{C}$. Fixed tissue was then processed using standard protocols and embedded in paraffin wax. Four-micrometre-thick sections were cut using a Thermo Scientific Microm HM325 microtome. Sectioned tissue was dried at $37^{\circ} \mathrm{C}$ overnight and then stored at $4{ }^{\circ} \mathrm{C}$.

Immunofluorescence using fresh frozen tissue. For immunofluorescence on fresh frozen tissue, cryosections were fixed in ice-cold acetone for $2 \mathrm{~min}$ and then air dried for $20 \mathrm{~min}$ at room temperature. Sections were then rinsed in PBS and incubated in $1 \% \mathrm{BSA} / \mathrm{PBS} / 0.1 \%$ Tween 20 for $30 \mathrm{~min}$ at room temperature to block nonspecific antibody binding. Primary antibody solutions diluted in PBS were then added at the appropriate dilution and incubated overnight at $4{ }^{\circ} \mathrm{C}$ or for $1 \mathrm{~h}$ at room temperature. The antibodies used and their dilutions were: anti-keratin 14 (rabbit polyclonal, Covance (PRB-155P-100), 1:500), anti-keratin 1 (rabbit polyclonal, Covance (AF 109), 1:500), anti-keratin 12 (goat polyclonal, Santa Cruz (sc-17101), 1:400), anti-ROCK2 (rabbit polyclonal, Abcam (ab71598), 1:100), anti-ROCK1 (rabbit IgG (EP786Y), Abcam, 1:100), anti-CD45 (rat IgG2b (30-F11), BD Biosciences, 1:200), anti- $\beta$-catenin (mouse IgG1 (14/beta-catenin), BD Biosciences, 1:50), anti-c-jun-phospho-S73 (rabbit polyclonal, Abcam (ab30620), 1:400), anti-FAK phospho-Y397 (rabbit polyclonal, Abcam (ab4803), 1:50), antiperiostin (mouse IgG (IC12), a gift from J. Huelesken, 1:1,000), anti-tenascin C (rat IgG1 (Mtn-12), Abcam, 1:400), anti-Ki67 (rabbit IgG (SP6), Abcam, 1:500).

Primary antibody solutions were then removed and sections were washed for $3 \times 5 \mathrm{~min}$ in PBS/0.1\% Tween 20. Sections were then incubated in the appropriate secondary antibody solutions diluted in PBS for $1 \mathrm{~h}$ at room temperature. The antibodies used and their dilutions were: donkey-anti-rabbit Alexa Fluor 488 (Molecular Probes, 1:800), donkey-anti-mouse Alexa Fluor 568 (Molecular Probes, 1:800), goat-anti-rat Alexa Fluor 568 (Molecular Probes, 1:1,000), donkey-anti-goat Alexa Fluor 568 (Molecular Probes, 1:600), donkey-anti-rabbit Alexa Fluor 647 (Invitrogen, 1:1,000), donkey-anti-rat Alexa Fluor 488 (Molecular Probes, 1:800), Donkey-anti-goat Alexa Fluor 488 (Molecular Probes, 1:800). DAPI (Sigma) was included in all secondary antibody solutions at a dilution of $1 / 4,000$. Secondary antibody solutions were then removed and sections were washed for $3 \times 5 \mathrm{~min}$ in PBS/0.1\% Tween 20. A final rinse was performed in PBS and stained sections were then mounted in DABCO (Sigma) anti-fade mounting medium.

Immunofluorescence using paraffin-embedded tissue. Paraffin-embedded tissue sections were dewaxed and rehydrated and were then washed for $2 \times 2 \mathrm{~min}$ in $\mathrm{dH} 2 \mathrm{O}$. Appropriate antigen retrieval was then performed depending on the primary antibody combinations used (see below for antigen retrieval conditions). Following antigen retrieval, sections were washed for $2 \times 2 \mathrm{~min}$ in PBS at room temperature and then blocked in $1 \% \mathrm{BSA} / \mathrm{PBS} / 0.1 \%$ Tween 20 for $30 \mathrm{~min}$ at room temperature. Primary antibody solutions diluted in $1 \% \mathrm{BSA} / \mathrm{PBS} / 0.1 \%$ Tween 20 were then added at the appropriate dilution and incubated overnight at $4{ }^{\circ} \mathrm{C}$ or room temperature for $90 \mathrm{~min}$. The antibodies used and their dilutions were: anti-keratin 1 (rabbit polyclonal, Covance (AF 109), 1:500), anti-keratin 12 (goat polyclonal, Santa Cruz (sc-17101), 1:400), anti- $\beta$-catenin (mouse IgG1 (14/betacatenin), BD Biosciences, 1:50), anti-c-jun-phospho-S73 (rabbit polyclonal, Abcam (ab30620), 1:400), anti-YAP/TAZ (mouse IgG2a (63.7), Santa Cruz, 1:200). Primary antibody solutions were then removed and sections were washed for $3 \times 5 \mathrm{~min}$ in PBS/0.1\% Tween 20. Secondary antibody solutions diluted in $1 \% \mathrm{BSA} / \mathrm{PBS} / 0.1 \%$ Tween 20 were then added at the appropriate dilution and incubated for $1 \mathrm{~h}$ at room temperature. The antibodies used and their dilutions were: donkey-antirabbit Alexa Fluor 488 (Molecular Probes, 1:800), donkey-anti-goat Alexa Fluor 568 (Molecular Probes, 1:600), donkey-anti-mouse Alexa Fluor 568 (Molecular Probes, 1:800). Secondary antibody solutions were then removed and sections were washed for $3 \times 5 \mathrm{~min}$ in $\mathrm{PBS} / 0.1 \%$ Tween 20 . A final rinse was performed in PBS and stained sections were then mounted in DABCO (Sigma) anti-fade mounting medium.

For YAP staining, a Mouse on Mouse blocking kit (Vectashield) was used and staining was performed according to the manufacturer's instructions.

Immunohistochemistry using paraffin-embedded tissue. For immunohistochemistry, antigen detection was performed using HRP-conjugated secondary antibodies combined with DAB (Sigma) revelation. Primary antibodies were as described for immunofluorescence. The staining procedure was as described for immunofluorescence with the addition of a peroxidase blocking step using $3 \% \mathrm{H}_{2} \mathrm{O}_{2}$. The secondary antibodies used were: donkey-anti-mouse IgG-HRP (Jackson, 1:100), goat-antirabbit IgG-HRP (Jackson, 1:100)

Antigen retrieval for immunofluorescence/immunohistochemistry on paraffin-embedded tissue. For detection of keratin 12, keratin 1, phosphoS73-cjun and $\beta$-catenin, sections were incubated in $10 \mathrm{mM}$ Tris/ $1 \mathrm{mM}$ EDTA/0.05\% Tween 20 (pH 9.0) for 20 min at $95^{\circ} \mathrm{C}$.
For detection of Ki67, sections were incubated in $10 \mathrm{mM}$ sodium citrate $/ 0.05 \%$ Tween 20 (pH 6.0) for $20 \mathrm{~min}$ at $95^{\circ} \mathrm{C}$.

Immunocytochemistry. Cells were rinsed in PBS, fixed in 4\% PFA for 15 min at room temperature and then washed twice with ice-cold PBS. Permeabilization was performed by incubating the cells in $\mathrm{PBS} / 0.25 \%$ Triton X-100 for $10 \mathrm{~min}$ at room temperature, followed by washing for $3 \times 5 \mathrm{~min}$ in PBS. Cells were then incubated in $1 \% \mathrm{BSA} / \mathrm{PBS} / 0.1 \%$ Tween 20 for $30 \mathrm{~min}$ at room temperature to block nonspecific antibody binding. Primary antibody solutions diluted in $1 \% \mathrm{BSA} / \mathrm{PBS} / 0.1 \%$ Tween 20 were then added and cells were incubated overnight at $4{ }^{\circ} \mathrm{C}$. The primary antibodies used and their dilutions were: anti-actin (mouse IgG1 (ACTNO5 (C4)), Abcam, 1:20), anti-keratin 12 (guinea pig polyclonal (AP09545SU-N), Acris, 1:200), antikeratin 1 (rabbit polyclonal, Covance (AF 109), 1:500), anti- $\beta$-catenin (mouse IgG1 (14/beta-catenin), BD Biosciences, 1:50), anti-YAP/TAZ (mouse IgG2a (63.7), Santa Cruz, 1:200), anti-Pax6 (rabbit polyclonal, Covance (PRB-278P-100), 1:500), anti-keratin 14 (rabbit polyclonal, Covance (PRB-155P-100), 1:500), anti-GFP (goat polyclonal, Abcam (ab6673), 1:400). Primary antibodies were removed and cells were washed for $3 \times 5 \mathrm{~min}$ in PBS. Secondary antibody solutions diluted in $1 \% \mathrm{BSA} / \mathrm{PBS} / 0.1 \%$ Tween 20 were then added and cells were incubated for $1 \mathrm{~h}$ at room temperature. The secondary antibodies used and their dilutions were: goatanti-mouse Alexa Fluor 488 (Molecular Probes, 1:1,000), donkey-anti-rabbit Alexa Fluor 647 (Molecular Probes, 1:1,000), donkey-anti-guinea pig biotin (Jackson, 1:200). For keratin 12 staining, an additional incubation in streptavidin Alexa Fluor 568 (Molecular Probes, 1:800) was required following incubation in the secondary antibody solutions. DAPI (Sigma) was included in all secondary antibody solutions. Cells were washed for $3 \times 5 \mathrm{~min}$ in PBS and then mounted in DABCO (Sigma; when cells were grown on coverslips) or stored in PBS.

Whole-mount immunofluorescence. Whole corneal tissue was dissected from the surrounding eye tissue and then incubated in $20 \mathrm{mM}$ EDTA/PBS (pH 7.4) at $37^{\circ} \mathrm{C}$ for $45 \mathrm{~min}$. The intact cornea was then placed epithelial side down onto Superfrost Plus histology slides (Thermo Scientific) and air dried for 3-5 min. Stromal tissue was then removed using forceps, leaving the intact corneal epithelium attached to the slide. The tissue was then fixed in ice-cold acetone for $2 \mathrm{~min}$ and then air dried for $20 \mathrm{~min}$ at room temperature. Immunofluorescent staining was performed using the protocols described for fresh frozen tissue.

Microscopy. Tiled images (fluorescent and bright-field) were acquired using a Leica DM5500 microscope fitted with Leica DFC 320 and DFC 350FX cameras. Confocal fluorescent images were acquired using a Zeiss LSM700 confocal microscope. Bright-field images were acquired using an Olympus AX70 microscope. Fluorescent images for immunocytochemistry experiments were acquired using an Olympus Cell XCELLENCE microscope fitted with a long-distance objective.

Image analysis. Quantification of $\beta$-catenin, K12, K1, pFAK, ROCK2, YAP/TAZ, tenascin $\mathrm{C}$ and periostin was performed using ImageJ software. For in vivo analyses, grid squares were used to specify regions corresponding to the limbus, peripheral $\mathrm{CE}$ and central CE. Mean fluorescence intensity was then measured bilaterally on 5 sections per cornea. Values were normalized to the fluorescence intensity measured in the conjunctiva of each section. For in vitro analyses, fluorescence intensity was measured on a per cell basis in 5 fields for each replicate.

Protein extraction and western blotting. Protein was extracted from whole cultured cells in RIPA buffer using standard protocols. Protein samples were resolved by SDS-PAGE ( $8 \%$ acrylamide) and then blotted onto PVDF membranes (Peqlab) by wet transfer using standard protocols. Unspecific interactions were blocked by incubation in 5\% milk (fat free) and then immunolabelling was performed using the following primary antibodies: anti-ROCK2 (rabbit polyclonal, Abcam (ab71598), 1:1,000), anti-actin (mouse IgG1 (ACTNO5 (C4)), Abcam, 1:2,000). Immunodetection was performed using the following HRP-conjugated secondary antibodies: anti-mouse IgG (GE Healthcare, 1:1,000), anti-rabbit IgG (GE Healthcare, 1:3,000). Revelation was performed using Pierce ECL Western Blotting Substrate (Thermo Scientific).

Quantitative real-time RT-PCR. Corneal epithelium was isolated from the underlying stroma by incubation in $20 \mathrm{mM}$ EDTA/PBS ( $\mathrm{pH}$ 7.4) containing RNA Later stabilization reagent for $45 \mathrm{~min}$ at $37^{\circ} \mathrm{C}$. Separated, intact corneal epithelium was then homogenized in Trizol reagent (Sigma) and total RNA was isolated according to the manufacturer's instructions and quantified using an ND100 NanoDrop spectrophotometer (NanoDrop Technologies). First-strand cDNA synthesis was then performed using Quantitect reverse transcription kit (Qiagen). Real-time PCR reactions were performed using SYBR Green and a 7900HT RealTime PCR System (both from Applied Biosystems) for 45 cycles. Specificity of the reactions was determined by melting curve analysis. Relative quantification was 
performed using the $2^{-\mathrm{DDCP}}$ method. $\alpha$-tubulin was used as the reference gene. Primer sequences were as follows: Notch1: Fwd 5'-CGCCCTTGCTCTGCCTA ACG-3', Rev 5'-TGCCACCATGGTCCACAACG-3'; Wnt1: Fwd 5'-ACAACAACG AGGCAGGGCG-3', Rev 5'-GGGGAGGGAGGCTTGTGC-3'; Wnt2: Fwd 5'-TGC CAAGGAGAGGAAAGGC-3', Rev 5'-TTCATGACTACCTGGATGGC-3'; Wnt $3 a$ : Fwd 5'-AACCGTCACAACAATGAGGC-3', Rev 5'-TTTCTCTACCACCATCTC CG-3'; Wnt4: Fwd 5'-TTCACAACAACGAGGCTGGC-3', Rev 5'-GGCTTGAACT GTGCATTCCG-3'; Wnt7b: Fwd 5'-TCTGCAACAAGATTCCTGGC-3', Rev 5'-G CGACGAGAAAAGTCGATGC-3'; Wnt10a: Fwd 5'-TCGGAACAAAGTCCCCT ACG-3', Rev 5'-TCCGACGGAAACGTTCTTCG-3'; Wnt10b: Fwd 5'-ACAACAA CAGGGTGGGACG-3', Rev 5'-TGGAACGCTCCAGAGTTGC-3'; GM-CSF: Fwd 5'-CCTTGGAAGCATGTAGAGGC-3', Rev 5'-ATATCTTCAGGCGGGTCTGC3'; IL-1 $\beta$ : Fwd 5'-CATTGTGGCTGTGGAGAAGC-3', Rev 5'-TGTCCATTGAGG TGGAGAGC-3'; S100A8: Fwd 5'-AACTGGAGAAGGCCTTGAGC-3', Rev 5'-CC ATCGCAAGGAACTCCTCG-3'; S100A9: Fwd 5'-CTTCTCAGATGGAGCGCAG C-3', Rev 5'-ATACACTCCTCAAAGCTCAGC-3'; IL-6: Fwd 5'-CACATGTTCTC TGGGAAATCG-3', Rev 5'-TCTTCATGTACTCCAGGTAGC-3'; GCSF: Fwd 5'-A AGTGCACTATGGTCAGGACG-3', Rev $5^{\prime}$-ACTGTGGAGCTGGCTTAGGC-3'; $\alpha$-tubulin: Fwd 5'-TCGTAGACCTGGAACCCACG-3', Rev 5'-GGTAAATGGAG AACTCCAGC- $3^{\prime}$.

Atomic force microscopy. Atomic force microscopy experiments were conducted with a Dimension Icon AFM scanner (Bruker) mounted on a custom-built support structure on top of an inverted optical microscope (IX71, Olympus). A $20-\mu \mathrm{m}-$ diameter bead (510-0166, VWR) was glued on the Lever D of a HYDRA-ALLG (AppNano) cantilever. The spring constant determined by the thermal noise method ${ }^{64}$ was $0.12 \mathrm{~N} \mathrm{~m}^{-1}$ and the same cantilever was used for all experiments. Force-volume measurements with 32 by 32 indentations over an area of $50 \mu \mathrm{m}$ by $50 \mu \mathrm{m}$ were taken at a rate of $1 \mathrm{~Hz}$ to a maximum force of $11.5 \mathrm{nN}$.

The elastic modulus on the approach direction of force curves was calculated assuming the Hertz contact model ${ }^{65}$. Subsequent analysis of elastic modulus maps was done using Matlab.
Fluorescence images were taken with a $20 \times$ objective (LUCPanFLN 20X, Olympus) and $2 \times$ magnifier in position with an iXon Ultra 897 (Andor) EMCCD camera. Illumination was achieved with a halogen lamp (U-HGLGPS, Olympus) and appropriate filter sets (MDF-GFP (Thorlabs) for K14-Alexa488, U-MNG2 (Olympus) for TenC-Alexa568, and F31-000 (AHF Analysentechnik AG) for DAPI). The relevant area was cropped and binned to a resolution of 32 by 32 pixels using standard image processing software to match the resolution obtained by AFM force-volume measurements. For wild-type samples, the image regions were segmented into regions of epithelium and stroma by applying a threshold on summed up intensity values from the fluorescent signals of K14-Alexa488 and DAPI, respectively. For mutant samples an additional intensity threshold on the fluorescent intensity of TenC-Alex568 was applied. Then ratios and standard deviations of the average elastic modulus values of the particular areas (epithelium and stroma) were calculated.

59. Huelsken, J., Vogel, R., Erdmann, B., Cotsarelis, G. \& Birchmeier, W. $\beta$-catenin controls hair follicle morphogenesis and stem cell differentiation in the skin. Cell 105, 533-545 (2001)

60. Indra, A. K. et al. Temporally-controlled site-specific mutagenesis in the basal layer of the epidermis: comparison of the recombinase activity of the tamoxifeninducible Cre-ER(T) and Cre-ER(T2) recombinases. Nucleic Acids Res. 27, 4324-4327 (1999).

61. Radtke, F. et al. Deficient T cell fate specification in mice with an induced inactivation of Notch1. Immunity 10, 547-558 (1999).

62. Behrens, A. et al. Impaired postnatal hepatocyte proliferation and liver regeneration in mice lacking c-jun in the liver. EMBO J. 21, 1782-1790 (2002).

63. Oshima, H., Rochat, A., Kedzia, C., Kobayashi, K. \& Barrandon, Y. Morphogenesis and renewal of hair follicles from adult multipotent stem cells. Cell 104, 233-245 (2001).

64. Hutter, J. L. \& Bechhoefer, J. Calibration of atomic-force microscope tips. Rev. Sci. Instrum. 64, 1868-1873 (1993).

65. Hertz, H. Ueber die Berührung fester elastischer Körper. J. für die reine und angewandte Math. 92, 156-171 (1881). 
a
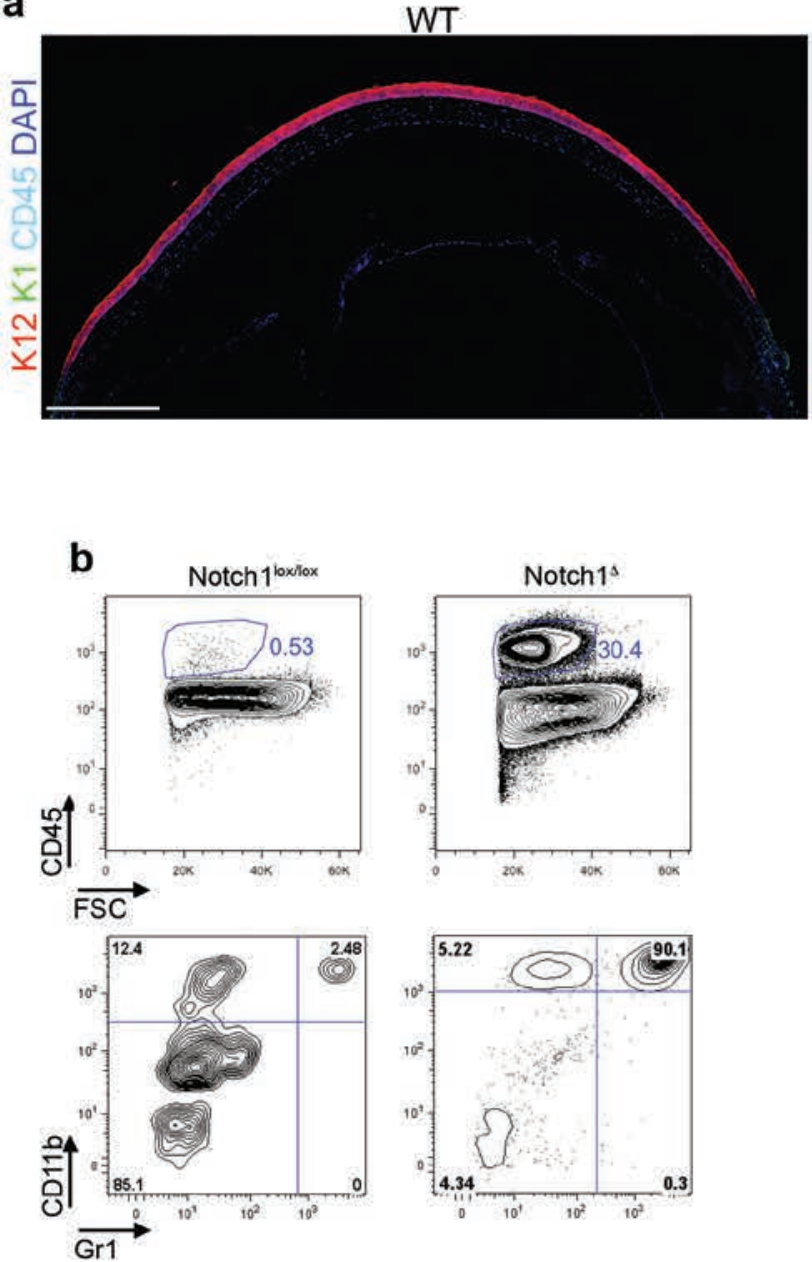

Supplementary Figure 1 Inflammation in Notch $1^{\Delta}$ mice following corneal injury. (a) Immunofluorescent staining for K12, K1 and CD45 in unwounded WT (Notch $1^{\text {lox/lox }}$ ) and Notch $1^{\Delta}$ corneas. Data are representative of 6 corneas over three independent experiments. (b) Flow cytometric analysis showing the proportion of total CD45+ cells (upper plots) and CD $45^{+} \mathrm{CD} 11 \mathrm{~b}^{+} \mathrm{Gr} 1^{+}$cells (lower plots) in WT (Notch $\left.1^{\text {lox/lox}}\right)$ and Notch $1^{\Delta}$ cornea 21 days after the repeated injury procedure (see Fig. 1a). Data are
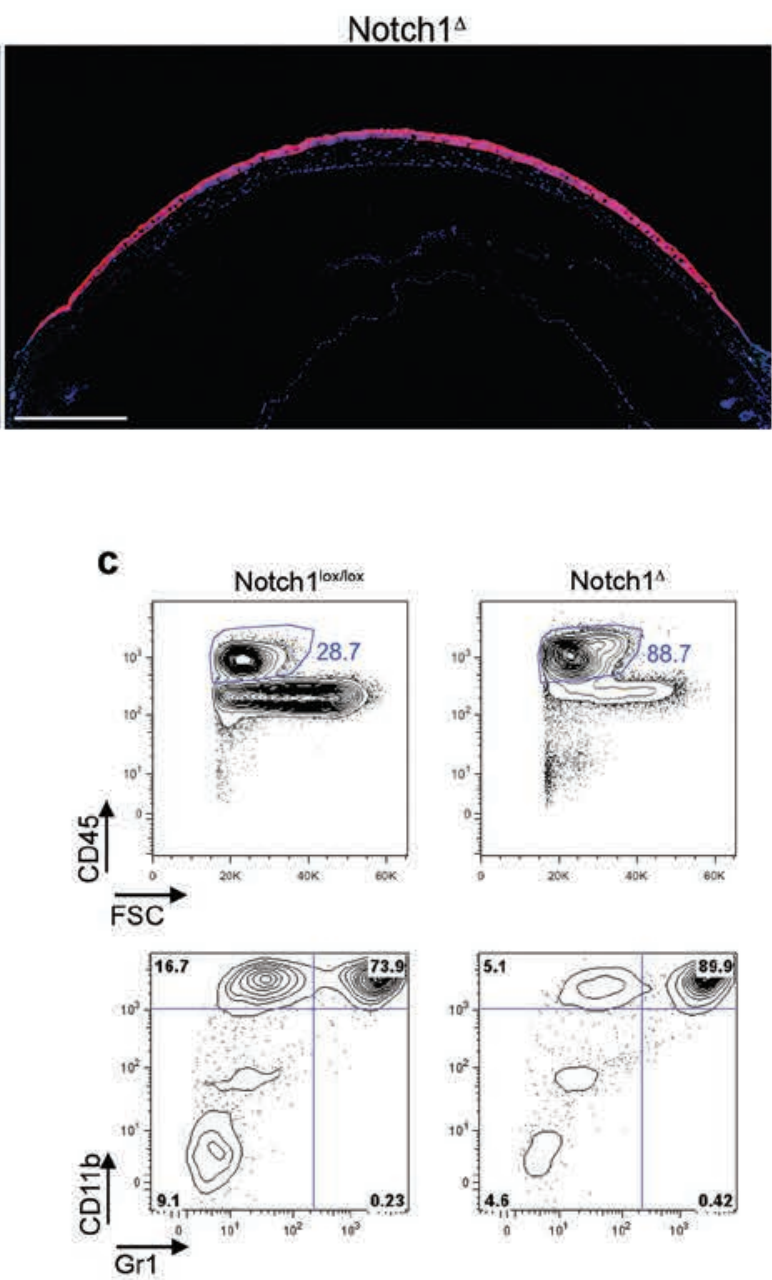

representative of 6 individual analyses performed on cells pooled from 4 corneas of each genotype over three independent experiments. (c) Flow cytometric analysis showing proportion of total CD45+ cells (upper plots) and $\mathrm{CD} 45^{+} \mathrm{CD} 11 \mathrm{~b}^{+} \mathrm{Gr} 1^{+}$cells (lower plots) in WT (Notch $\left.1^{\text {lox/lox}}\right)$ and Notch $1^{4}$ cornea 24 hours after a single injury. Data are representative of 6 individual analyses performed on cells pooled from 4 corneas of each genotype over three independent experiments. Scale bars represent $500 \mu \mathrm{m}$. 

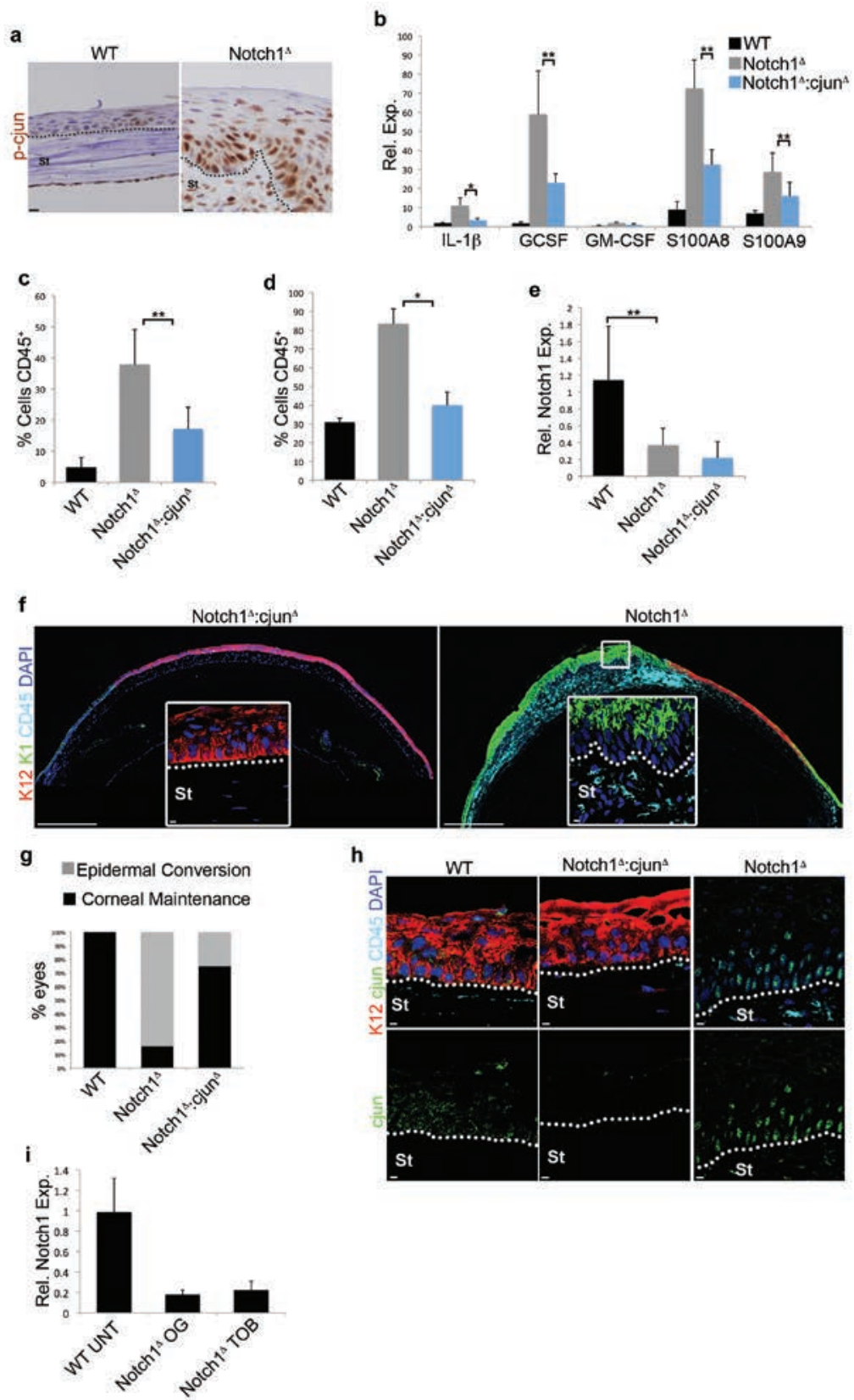

Supplementary Figure 2 Chronic inflammation is associated with CSCM in Notch $1^{\Delta}$ mice. (a) Immunohistochemistry for ${ }^{p S 73}$ cjun following repeated corneal injury. (b) QRT-PCR analysis for the indicated cytokines in WT (Notch $1^{\text {lox/lox }}$ : cjun ${ }^{\text {lox/lox }}$, Notch $1^{\Delta}$ and Notch $1^{\Delta}:$ cjun $^{\Delta}$ corneal epithelial cells 24 hours after a single corneal injury ( $n=6$ biological replicates for each genotype over three independent experiments. Each replicate consists of corneal epithelial tissue pooled from 6 corneas isolated from 3 mice of each genotype). Data are expressed relative to the expression in WT unwounded corneal epithelial cells. (c,d) Quantification of the proportion of CD45+ cells in WT (Notch $1^{\text {lox/lox }}$ : $j u n^{\text {lox/lox }}$ ), Notch $1^{\Delta}$ and Notch $1^{\Delta}:$ cjun $^{\Delta}$ cornea 21 days after repeated corneal injury (c) or 24 hours after a single corneal injury (d). Proportions were measured by performing flow cytometry on dissociated corneas. ( $n=6$ biological replicates for each genotype over three independent experiments. Each replicate consists of cells pooled from 4 corneas isolated from 2 mice of each genotype). (e) QRT-PCR analysis of Notch1 in the corneal epithelium of WT (Notch1 1ox/lox:cjun ${ }^{\text {lox/lox }}$ ), Notch $1^{\Delta}:$ cjun $^{\Delta}$ and Notch $1^{\Delta}$ mice following repeated injury ( $n=6$ biological replicates for each genotype over three independent experiments. Each replicate consists of corneal epithelial tissue pooled from 6 corneas isolated from 3 mice of each genotype). ( $f$ ) Representative immunofluorescent staining for K12, K1 and CD45 in Notch $1^{\Delta}$ :cjun ${ }^{\Delta}$ and Notch $1^{\Delta}$ corneas following repeated corneal injury. Large panels are low magnification tiled images. White outlined insets show high magnification images of the indicated regions. (g) Proportion of WT (Notch $1^{\text {lox }}$ ${ }^{l o x}:$ cjun $^{\text {lox/lox }}$ ( $\mathrm{n}=10$ corneas), Notch ${ }^{\Delta}$ ( $\mathrm{n}=20$ corneas) and Notch $1^{\Delta}:$ cjun $^{\Delta}$ ( $n=16$ corneas) corneal tissue exhibiting corneal or epidermal identity after repeated corneal injury. (h) Immunfluorescent staining for $\mathrm{K} 12, \mathrm{~K} 1$, cjun (pS73) and CD45 on corneal tissue isolated from WT (Notch1lox/lox:cjun lox/ lox), Notch $1^{\Delta}:$ cjun $^{\Delta}$ and Notch $1^{\Delta}$ mice after repeated corneal injury. Data are representative of 16 corneas. (i) QRT-PCR analysis of Notch 1 in the corneal epithelium of WT (Notch $1^{\text {lox/lox }}$ ) and Notch ${ }^{\Delta}$ mice treated with ophthalmic lubricating gel or Tobradex following repeated injury $(n=6$ biological replicates for each treatment over three independent experiments. Each replicate consists of corneal epithelial tissue isolated during corneal wounding of a single eye). Scale bars represent $500 \mu \mathrm{m}$ on tiled images and $5 \mu \mathrm{m}$ on all other images. St - Stroma. * $\mathrm{P}<0.01,{ }^{*} * \mathrm{P}<0.05$ (unpaired, two tailed t-tests). Error bars represent standard deviation. 
a
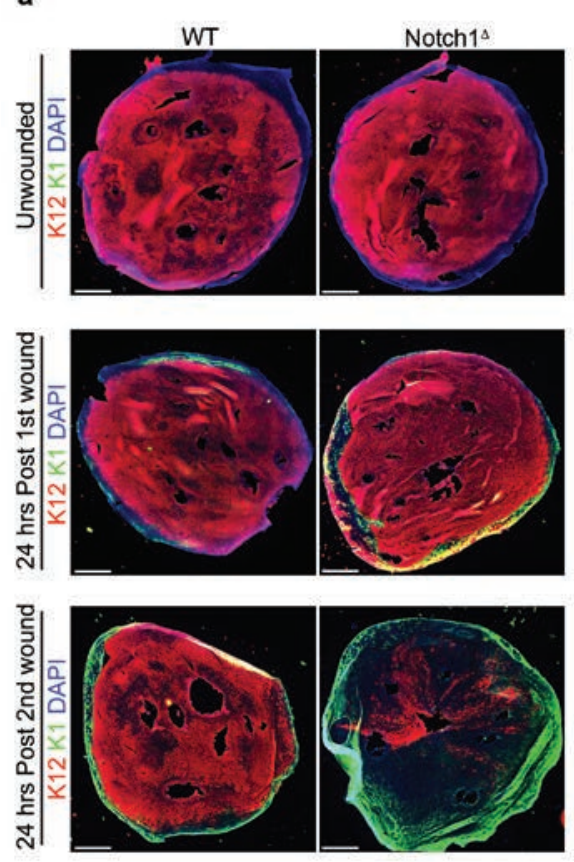

b

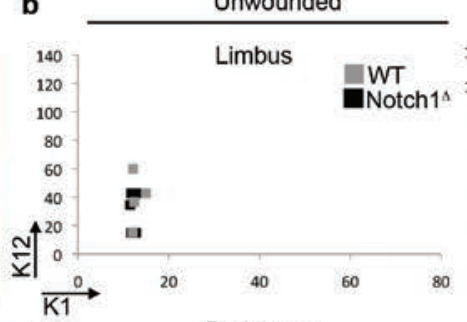

Periphery
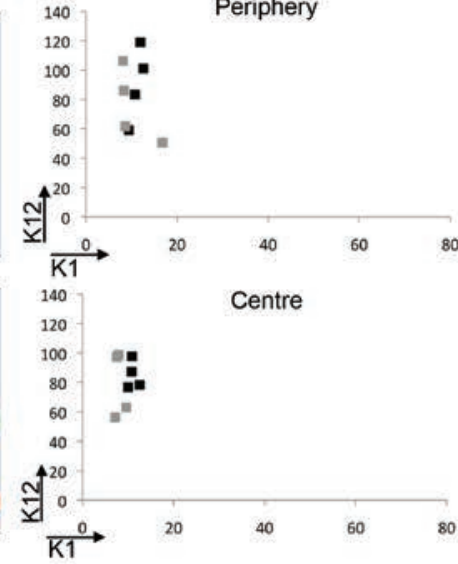

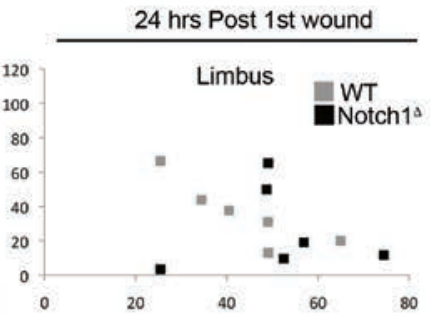

Periphery
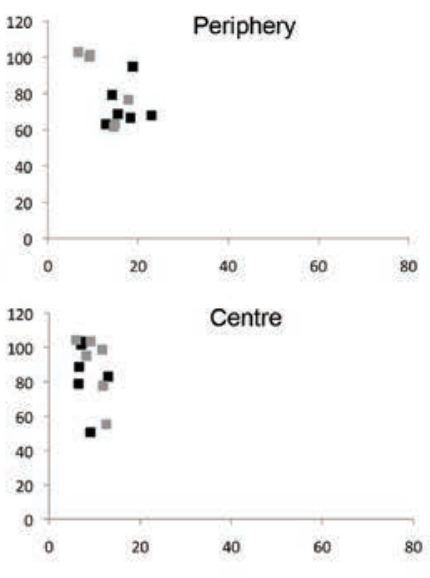
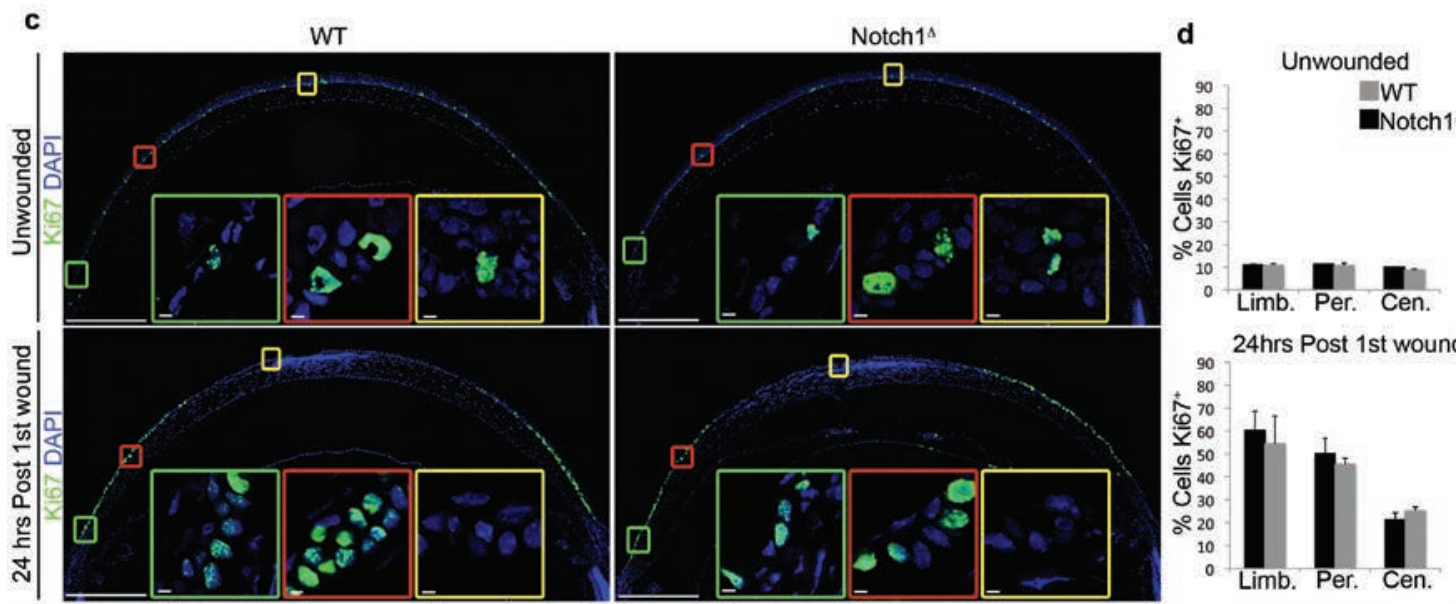

24hrs Post 1st wound
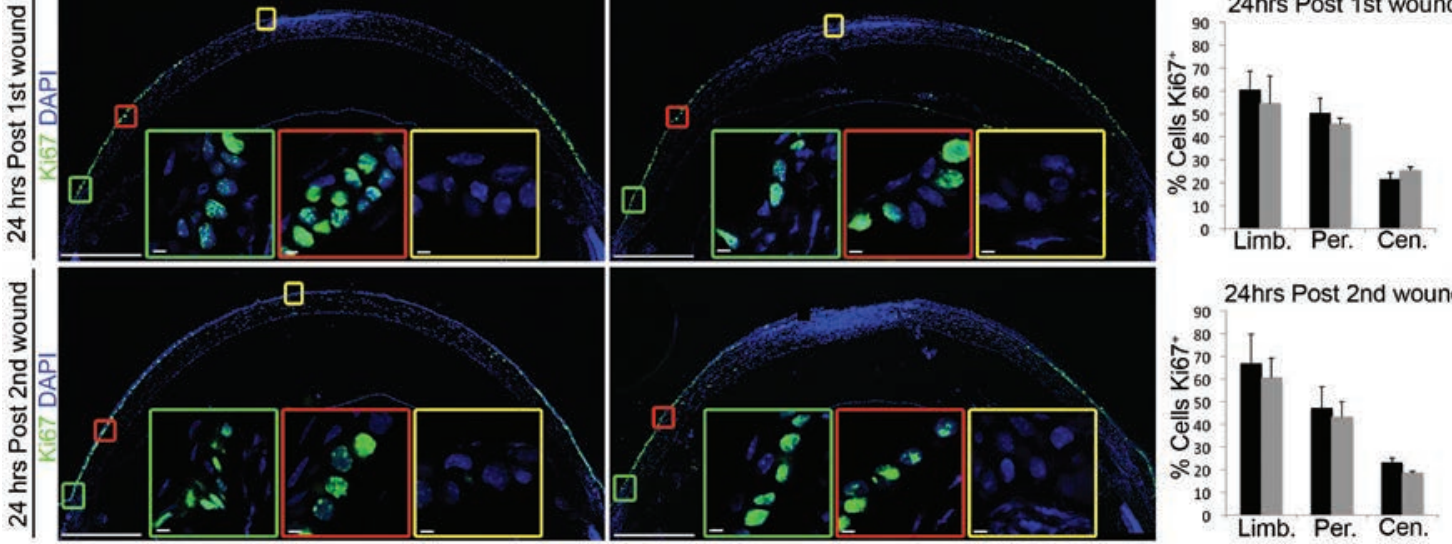

Supplementary Figure 3 Spatial and temporal kinetics of CSCM (a) Representative immunofluorescent staining for $\mathrm{K} 12$ and $\mathrm{K} 1$ on wholemount corneal epithelial tissue isolated from WT and Notch $1^{\Delta}$ corneas. Upper panels show unwounded corneas, middle panels show corneal epithelial tissue 24 hours after the first injury, lower panels show corneal epithelial tissue 24 hours after the second injury. Data are representative of 6 corneal wholemounts over three independent experiments. (b) XY scatter plots showing $\mathrm{K} 12$ and $\mathrm{K} 1$ expression in limbus, peripheral cornea and central cornea in unwounded corneas and in corneas isolated 24 hours after the second corneal injury. Each data point represents mean fluorescence intensity measured from an individual cornea. Grey boxes $=$ WT $\left(\right.$ Notch $\left.1^{\text {lox/lox }}\right)$, black boxes $=\operatorname{Notch}^{\Delta}(n=6$ corneas for each genotype over three independent experiments). (c) Immunofluorescent staining for Ki67 on WT and Notch ${ }^{\Delta}$ corneas. Data are representative of 6 corneas. Upper panels show unwounded corneas, middle panels show corneal tissue 24 hours after the first injury, lower panels show corneal tissue 24 hours after the second injury. Large panels are low magnification tiled images. Insets outlined in green, red and yellow show high magnification images of the limbus, peripheral cornea and central cornea respectively. (d) Quantification of the proportion of Ki67+ cells in the limbus, peripheral cornea and central cornea in unwounded corneas (upper panels), corneas 24 hours after the first injury (middle panels) and corneas 24 hours after the second injury (lower panels). Black boxes = WT $\left(\right.$ Notch $\left.1^{\text {lox/lox }}\right)$, grey boxes $=\operatorname{Notch}^{\Delta}(n=6$ corneas for each genotype over three independent experiments). Scale bars represent $500 \mu \mathrm{m}$ on tiled images and $5 \mu \mathrm{m}$ on all other images. St - Stroma, Limb - limbus, Per Periphery, Cen - Centre. Error bars represent standard deviation. 
a

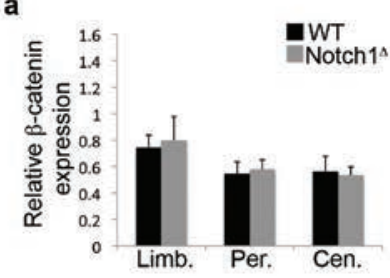

C

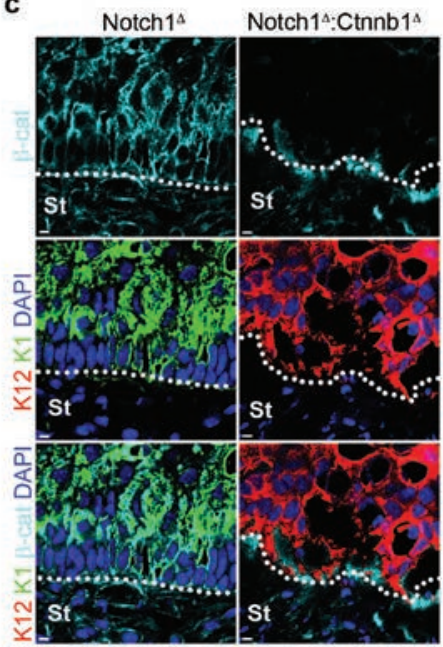

b 1.6$] \quad$ WT

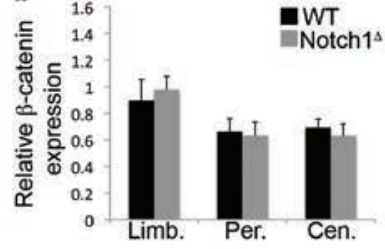

d<smiles>[Te]=[W]</smiles>

Ctnnb1 ${ }^{\triangle \mathrm{EEx}}$

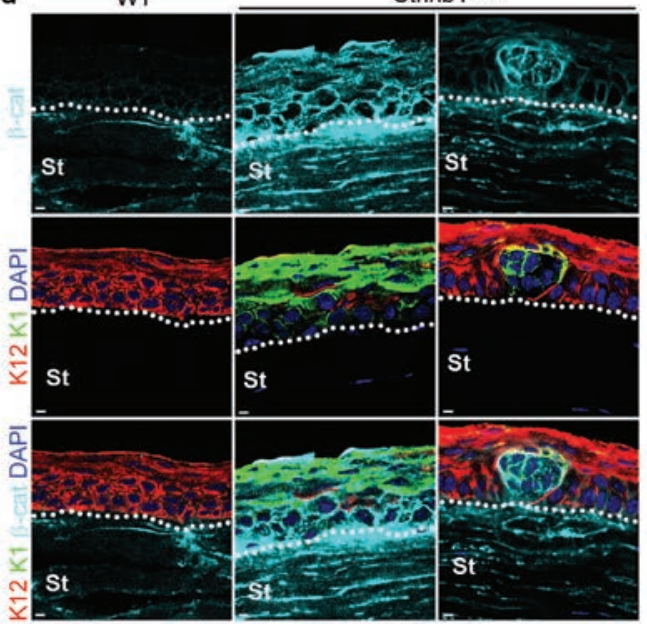

K12 K1 DAPI
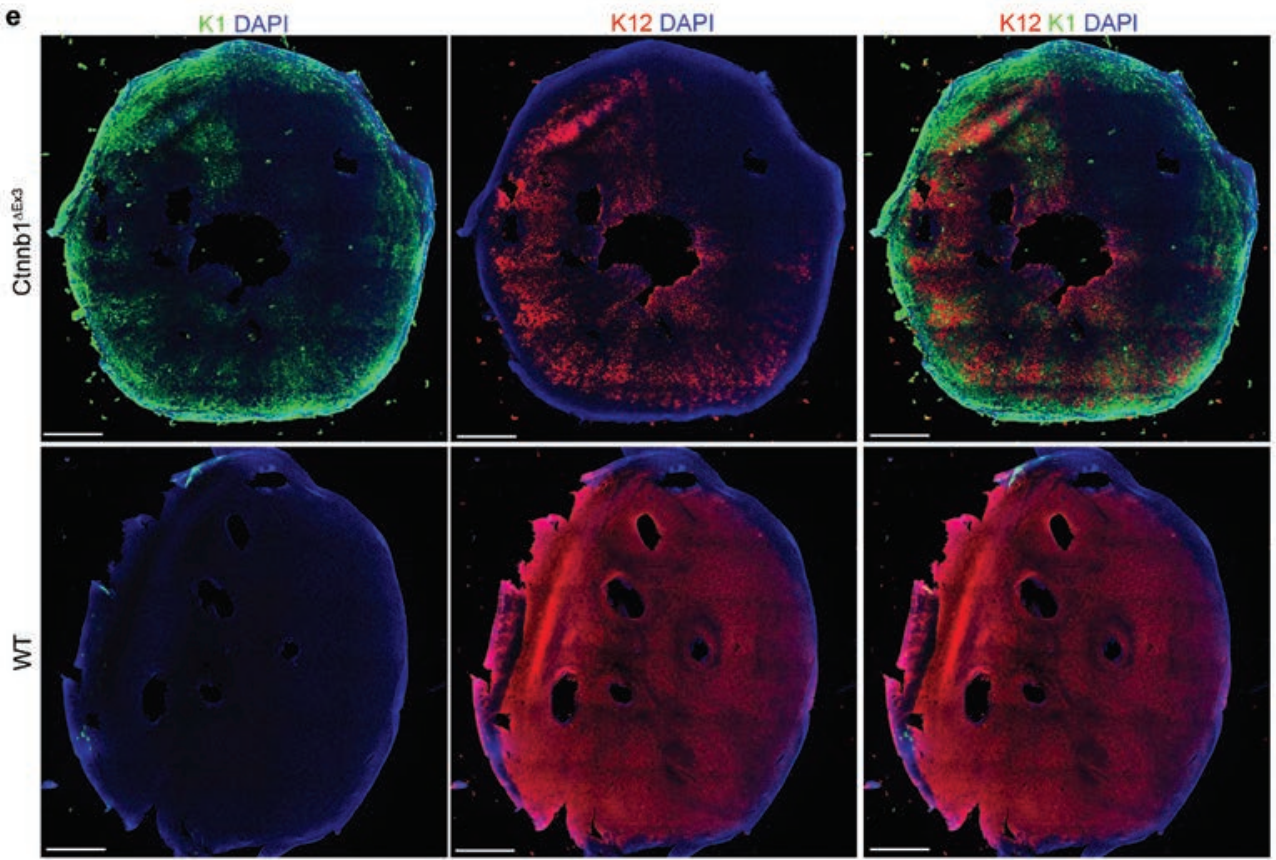

Supplementary Figure 4 Elevated $\beta$-catenin is necessary and sufficient to induce CSCM. (a-b) Quantification of relative $\beta$-catenin expression in limbus, peripheral cornea and central cornea in unwounded eyes (a) and 24 hours after the first injury (b). Black bars $=$ WT (Notch $\left.1^{\text {lox/lox }}\right)$, grey bars $=\operatorname{Notch~}^{\Delta}$ ( $n=6$ corneas for each genotype over three independent experiments). Values for expression levels are relative values normalised to the expression level in the conjunctiva of each sample, determined by mean fluorescence intensity. (c) Immunofluorescent staining for $\beta$-catenin, $\mathrm{K} 12$ and $\mathrm{K} 1$ on Notch $1^{\Delta}$ and Notch $1^{\Delta}$ :Ctnnb $1^{\Delta}$ corneas after repeated injury. Data are representative of 8 corneas for each genotype over 3 independent experiments. (d) Immunofluorescent staining for $\beta$-catenin, K12 and K1 on WT (Ctnnb1 $\left.1^{\operatorname{lox}(E \times 3) / / o x(E \times 3)}\right)$ and Ctnnb $1^{\Delta E \times 3}$ corneas 7 days after a single injury. Data are representative of 8 WT corneas and $10 \mathrm{Ctnnb}^{\Delta E \times 3}$ corneas over 4 independent experiments. (e) Immunofluorescent staining for K12 and K1 on wholemount corneal epithelial tissue isolated from or WT (Ctnnb1 lox(ex3)/lox(ex3) $)$ or Ctnnb1 $1^{\Delta E \times 3}$ corneas 7 days after a single corneal injury. Data are representative of 6 WT and 8 Ctnnb $1^{\Delta E x 3}$ corneas over 3 independent experiments. Scale bars represent $500 \mu \mathrm{m}$ on tiled wholemount images and $5 \mathrm{~mm}$ on all other images. Error bars represent standard deviation. 

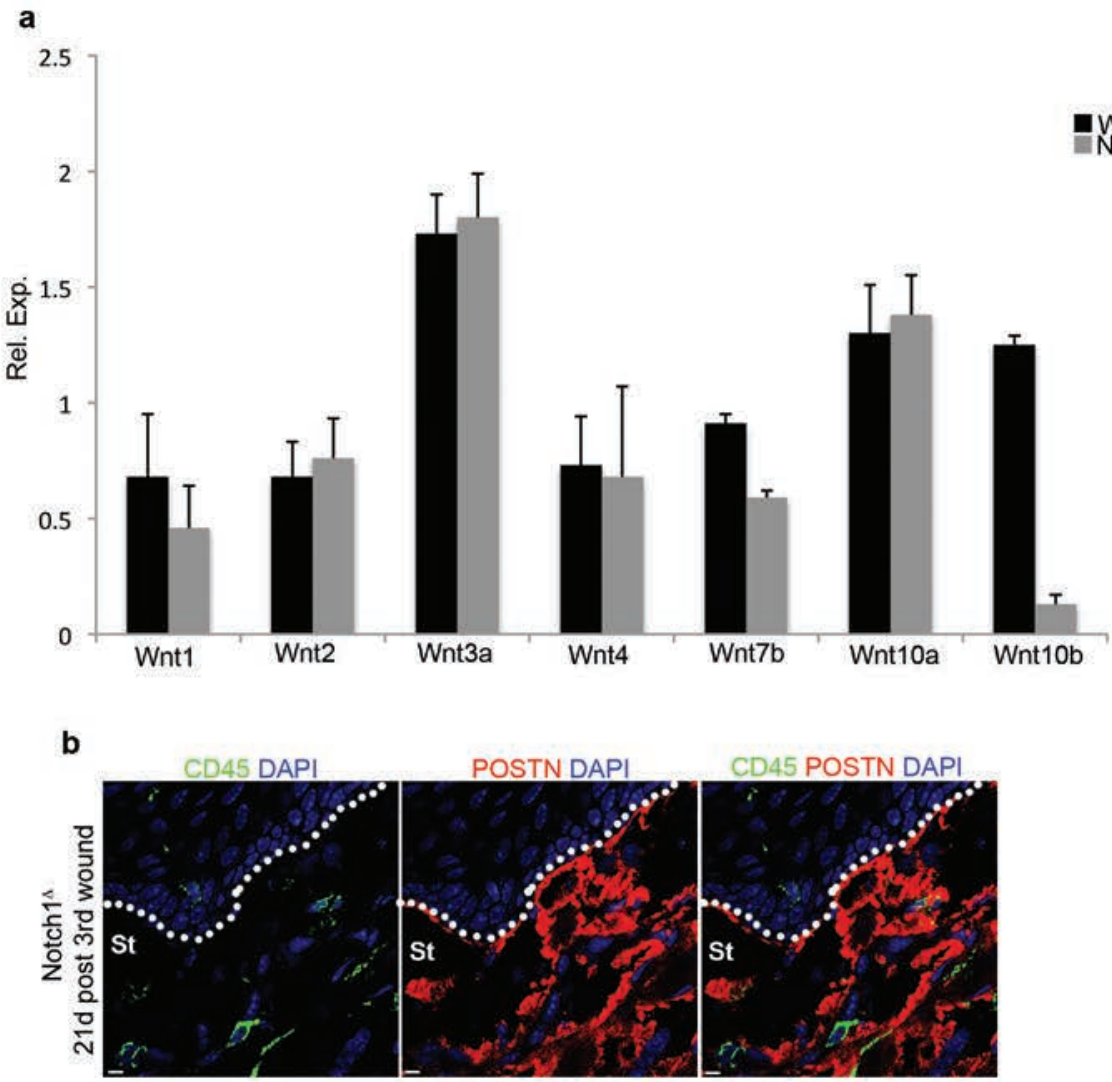

C Notch $1^{\triangle} 21 \mathrm{~d}$ post 3rd wound

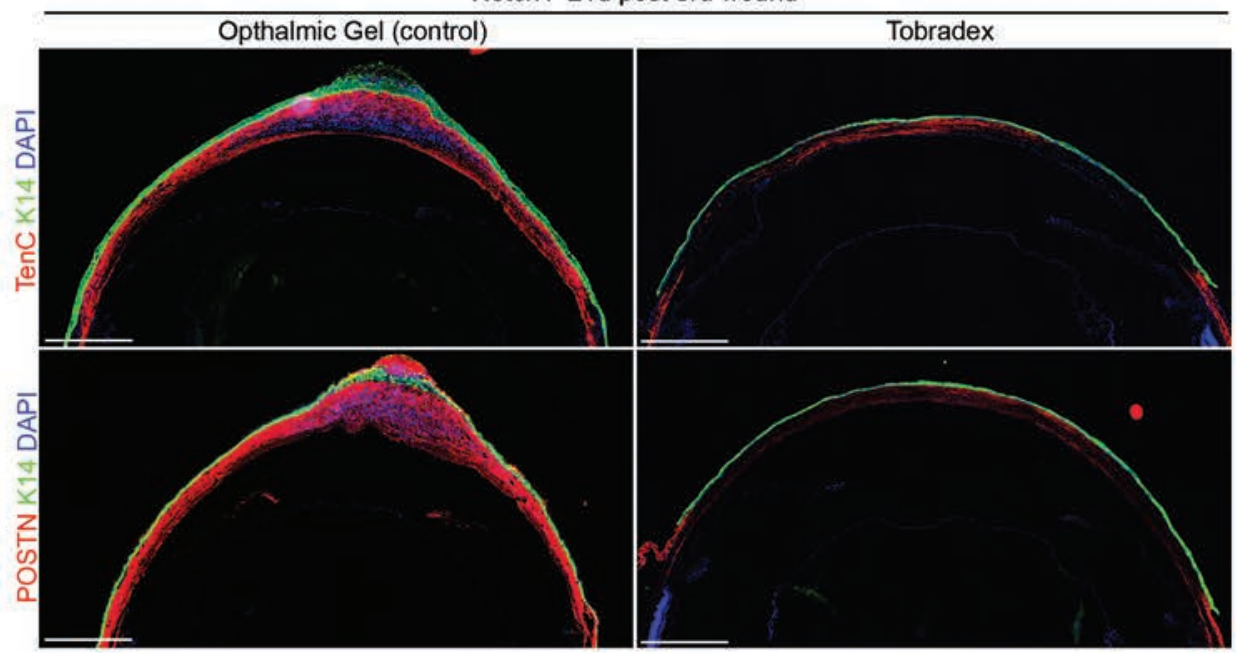

Supplementary Figure 5 Inflammation induced CSCM is associated with increased ECM deposition in the corneal stroma. (a) QRT-PCR analysis of Wnt ligands that exhibit expression in corneal tissue after repeated injury. Analysis was performed on whole corneal tissue isolated from WT (Notch $1^{\text {lox } / l o x}$ ) and Notch $1^{\Delta}$ mice 21 days after the $3^{\text {rd }}$ corneal injury $(n=5$ biological replicates for each genotype over four independent experiments. Each replicate consists of corneal epithelial tissue pooled from 4 corneas isolated from 2 mice of each genotype). Data are expressed relative to the expression in whole corneal tissue isolated from unwounded mice. Error bars represent standard deviation. (b) Immunofluorescence for Periostin, CD45 and DAPI on chronically inflamed Notch $1^{\Delta}$ corneal tissue after repeated injury. Single fluorescent images and merges are shown as indicated. Data are representative of 4 corneas analysed over two independent experiments. Scale bars represent $5 \mathrm{~mm}$. (c) Immunofluorescent staining for $\mathrm{K} 14$ and Tenascin C (upper panels) or K14 and Periostin (lower panels) in Notch ${ }^{4}$ mice treated with either ophthalmic gel (control) or Tobradex during the repeated injury procedure. Data are representative of 6 corneas analysed over three independent experiments. Scale bars represent $500 \mu \mathrm{m}$. 
a
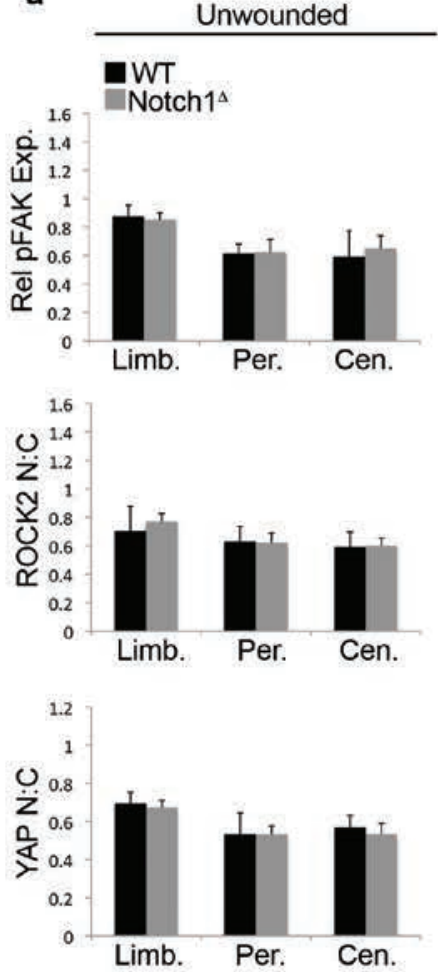

b
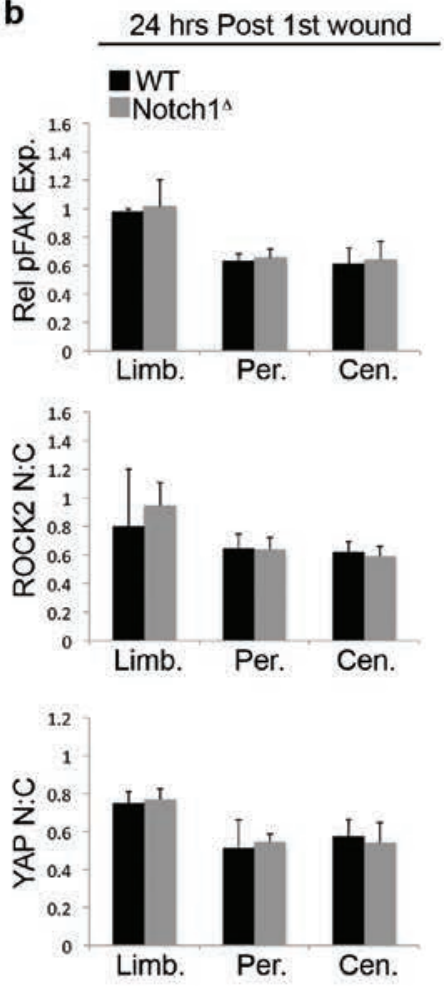

c

Notch $1^{\triangleleft} 21 \mathrm{~d}$ post 3 rd wound
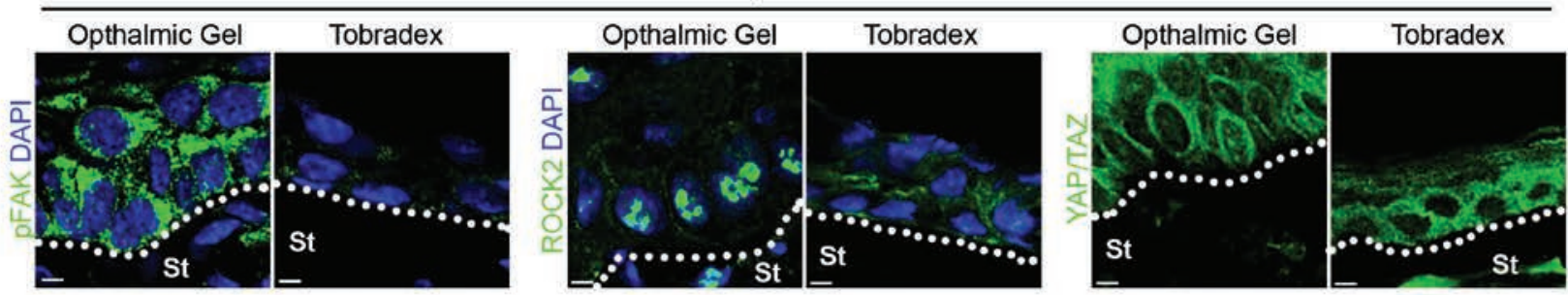

Supplementary Figure $6 \mathrm{CSCM}$ is associated with mechanotransduction. $(a, b)$ Quantification of FAK phosphorylation (upper panels) and nuclear:cytoplasmic ratio of ROCK2 (middle panels) and YAP/TAZ (lower panels) in the limbus, peripheral cornea and central cornea isolated from unwounded eyes (a) or eyes isolated 24 hours after the first corneal injury (b). Values for expression levels are relative values normalised to the expression level in the conjunctiva of each sample, determined by mean fluorescence intensity. Black bars $=$ WT $\left(\right.$ Notch $\left.1^{\text {lox/lox }}\right)$, grey bars $=$ Notch $1^{\Delta}$ ( $n=6$ corneas for each genotype over three independent experiments). Error bars represent standard deviation. (c) Immunofluorescence for PFAK, ROCK2 and YAP/TAZ on corneal tissue isolated from Notch $1^{\Delta}$ mice treated with ophthalmic gel (control) or Tobradex during the repeated injury procedure. Data are representative of 6 corneas for each treatment analysed over three independent experiments. Scale bars represent $5 \mu \mathrm{m}$. 

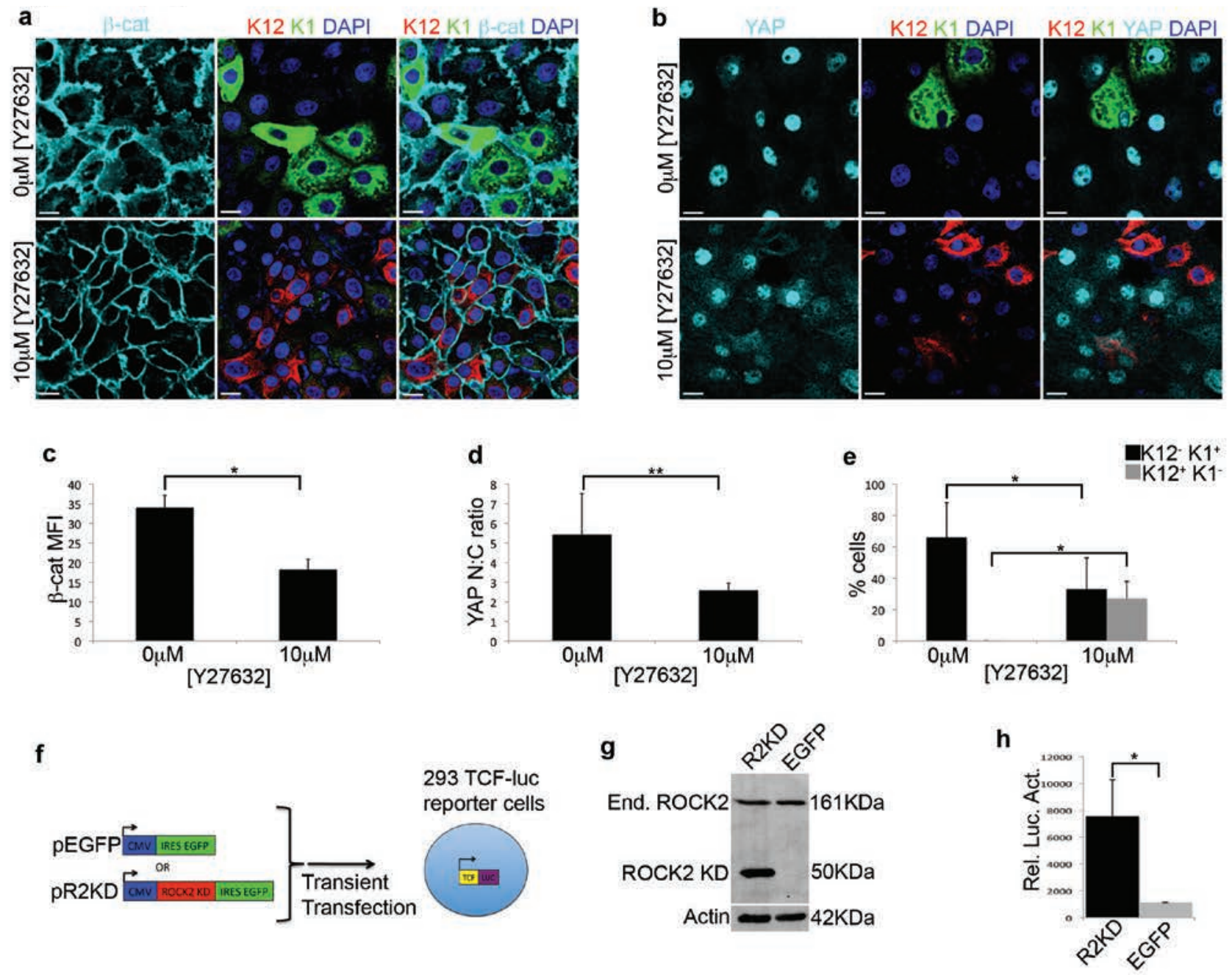

Supplementary Figure 7 Manipulation of mechanotransduction affects $\beta$-catenin expression in corneal epithelial cells. (a,b) Immunofluorescence for $\beta$-catenin, K12 and K1 (a) or YAP/TAZ, K12 and K1 (b) on PCESCs cultured on stiff (glass - >2GPa) substrates and maintained in vehicle (PBS - upper panels) or 10 $\mu \mathrm{M}$ Y27632 (lower panels). Data are representative of 8 individual cultures for each condition. (c-e) Quantification of $\beta$-catenin expression (c), YAP/TAZ nuclear:cytoplasmic ratio (d) and the proportion of $\mathrm{K} 12^{+} \mathrm{K} 1^{-}$and $\mathrm{K} 12^{-} \mathrm{K} 1^{+}$cells (e) in PCESCs maintained in vehicle (PBS) or

$10 \mu \mathrm{M}$ Y27632 ( $\mathrm{n}=8$ individual cultures over four independent experiments). For (c) $\beta$-catenin expression is determined by mean fluorescence intensity. (f) Schematic depicting the transfection of TCF-Luc 293 T cells with pR2KD or pEGFP. (g) Western blot performed on TCF-Luc 293T cells 48 hours after transfection with pR2KD or pEGFP. (h) Relative luciferase activity in TCF-Luc 293 T cells 48 hours after transfection ( $n=6$ individual transfections over three independent experiments). ${ }^{*} P<0.01,{ }^{* *} P<0.05$, (unpaired, two tailed t-tests). Error bars represent standard deviation. Scale bars represent $20 \mu \mathrm{m}$. 